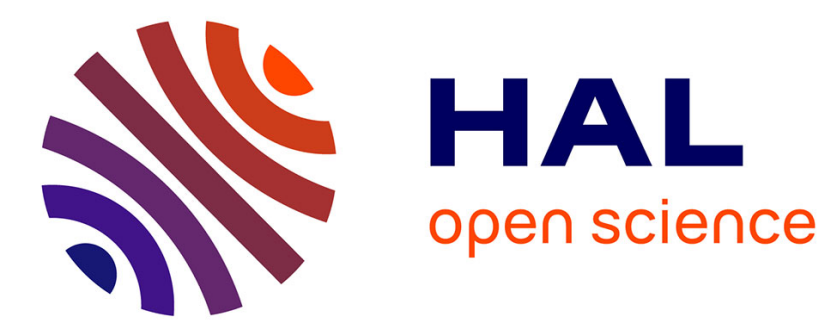

\title{
Local Proportional-Integral Boundary Feedback Stabilization for Quasilinear Hyperbolic Systems of Balance Laws
}

\author{
Liguo Zhang, Christophe Prieur, Junfei Qiao
}

\section{- To cite this version:}

Liguo Zhang, Christophe Prieur, Junfei Qiao. Local Proportional-Integral Boundary Feedback Stabilization for Quasilinear Hyperbolic Systems of Balance Laws. SIAM Journal on Control and Optimization, 2020, 58 (4), pp.2143-2170. 10.1137/18M1214883 . hal-02956431

\section{HAL Id: hal-02956431 \\ https://hal.science/hal-02956431}

Submitted on 2 Oct 2020

HAL is a multi-disciplinary open access archive for the deposit and dissemination of scientific research documents, whether they are published or not. The documents may come from teaching and research institutions in France or abroad, or from public or private research centers.
L'archive ouverte pluridisciplinaire HAL, est destinée au dépôt et à la diffusion de documents scientifiques de niveau recherche, publiés ou non, émanant des établissements d'enseignement et de recherche français ou étrangers, des laboratoires publics ou privés. 


\title{
LOCAL PI BOUNDARY FEEDBACK STABILIZATION FOR QUASILINEAR HYPERBOLIC SYSTEMS OF BALANCE LAWS*
}

\author{
LIGUO ZHANG ${ }^{\dagger}$, CHRISTOPHE PRIEUR ${ }^{\ddagger}$, AND JUNFEI QIAO $^{\dagger}$
}

\begin{abstract}
The proportional-integral boundary stabilization of nonlinear hyperbolic systems of balance laws is investigated for the $H^{2}$-norm, in which the control and output measurements are all located at the boundaries. The boundary conditions of the system are subject to unknown constant disturbances. The induced closed-loop system is proven to be locally exponentially stable with respect to the steady states. To this end, a set of matrix inequalities is given by constructing a new Lyapunov function as a weighed $H^{2}$-norm of the classical Cauchy solution and the integral of boundary output. Furthermore, the traffic flow dynamics of a freeway section are modeled with Aw-Rascle-Zhang model. To stabilize the oscillations of traffic demand, a local PI boundary feedback controller is designed with the integration of the on-ramp metering and the variable speed limit control. The exponential convergence of the nonlinear traffic flow dynamics in $H^{2}$ sense is achieved and validated with simulations.
\end{abstract}

Key words. Quasilinear hyperbolic systems, Balance laws, PI boundary feedback stabilization, Lyapunov function, ARZ traffic flow model.

AMS subject classifications. 93C20, 93D15, 35Q53

1. Introduction. Hyperbolic systems of balance laws have been extensively used to represent the fundamental dynamics of many physical processes. An interesting example is Aw-Rascle equation in [1], or Aw-Rascle-Zhang equation in [34, in which the variation of the average density and speed of vehicles over a road is balanced by its flux through the boundaries and its reaction of drivers inside the road. Other typical examples include Saint-Venant equation in [12] for open channels, Euler equation in [18 for gas pipes, telegrapher equations in [13] for electrical transmission lines, and Kac-Goldstein equation in [24] for chemotaxis.

Boundary feedback control is central for stabilization of the hyperbolic systems where the control units and measurements are all located at boundaries of the domain. The initial result for the asymptotic stability is presented by Greenberg and Li in [14. Recently, the Lyapunov technique is proposed in [7] for the exponential stability of nonlinear hyperbolic system of conservation laws. Following the static boundary feedback control law, many results have been established in [19, 37, 10, and [17] for the $L^{2}$ or $H^{2}$ topology. The stabilization of the isothermal Euler equations to non-constant states that depend on the space variable has been solved in [18]. Inspired from [7], we develop a dynamic proportional-integral (PI) boundary feedback control for stabilization of the general nonlinear hyperbolic system of balance laws.

As it is well-known that PI feedback control is one of the most widely used method in engineering since its advantage is to cancel the forced oscillations. One disadvantage of it is that, in some sense, it might damage the stability. Frequency domain is the most used approach to prove the stability of the linear hyperbolic system. An explicit necessary and sufficient condition is proposed in [5] for the linearized density-flow system by using Laplace transformation. Similar results are also given by combining the operator and semi-group approach in [32, 29. Backstepping is used in 22] to elaborate the PI-based trajectory tracking for the linear hyperbolic system. It is also interesting to note that PI observer is proposed in 2] for the hydraulic cylinder system by using the backstepping method. Moreover, Lyapunov approach is considered in [11] and 31] for the linear hyperbolic conservation laws, and in [16] for the wave equation. The significant advantage of this direct method is that it can be extended to deal with the nonlinear systems besides offers the tractable method to design the control law.

In [30, the integral controller is designed for a scalar nonlinear hyperbolic system of conservation laws, in which the output measurement is regulated to the desired reference but the equilibrium of the system is unknown. In our paper, the final desired state of the nonlinear partial differential equations (PDE) is fixed in advance although the boundary conditions are corrupted by the unknown disturbance. We consider the PI boundary stabilization of nonlinear hyperbolic system of balance laws and prove the local exponential stability for the $H^{2}$-norm. A new Lyapunov function as a weighed $H^{2}$-norm of the classical Cauchy solution and the

*This work is supported by the National Natural Science Foundation of China (NSFC, grant No. 61873007), and the Beijing Natural Science Foundation (grant No. 1182001).

${ }^{\dagger}$ Key Laboratory of Computational Intelligence and Intelligent Systems, Faculty of Information Technology, Beijing University of Technology, 100124, Beijing, China. E-mail: zhangliguo@bjut.edu.cn; junfeiq@bjut.edu.cn

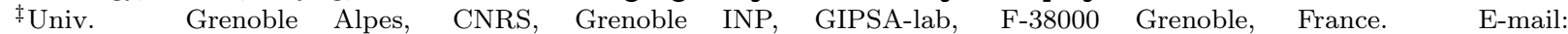
christophe.prieur@gipsa-lab.fr 
integral of the boundary output is constructed. The time derivative of this Lyapunov function can be made strictly negative definite by an appropriate choice of boundary gains to meet a set of matrix inequalities.

Aw-Rascle-Zhang (ARZ) model, compared to the scalar LWR model [23] and [27, is a second-order hyperbolic system with developed speed equation to reveal the anisotropic property of the macroscopic traffic flow dynamics [9. Adopting boundary feedback control is natural for the system since available control signals for on-ramp metering or VMSs (Variable Message Signs) for regulating vehicle speed usually located at boundaries of a road. In [6], the spectral analysis is applied to design the boundary control laws for the linearized ARZ model. An integrated on-ramping metering and variable speed limit control is proposed in [35] by investigating stabilization of positive hyperbolic systems. For the Markov jumped hyperbolic systems, [36] develops the stochastic stability with application to ARZ model including one free-flow and one congested modes. Recently, boundary feedback control laws are developed in [33 by using backstepping to reduce the stop-and-go oscillations for congested traffic.

This paper further studies the problem of traffic flow control via PDEs technique as the traffic demand of the road is uncertain. As the flow-rate of the driving-in vehicles through the upstream boundary is affected by the fluctuating traffic demand, the developed PI boundary feedback laws can be used to suppress the uncertainties and stabilize the traffic parameters (the vehicle density and speed) at the desired states by using the integrated on-ramp metering and variable speed control.

The rest of the paper is organized as follows. The statement of the PI boundary feedback control for the nonlinear hyperbolic system of balance laws and the main result are given in Section 2 . In Section 3 we presents the proof of the main result by using the Lyapunov techniques in the $H^{2}$ topology. Section 4 is devoted to the boundary feedback stabilization of a freeway section with the uncertain traffic demand based on the nonlinear ARZ equation. Some concluding remarks and possible further research lines are presented in Section 5.

Notation: $\mathbb{R}^{n}$ and $\mathbb{R}^{n \times n}$ denote the set of $n$-order vectors and $n$-order matrices. For a matrix $A$, $A>0$ means that $A$ is a symmetric positive definite matrix, $A^{\top}$ is the transpose, and for a partitioned symmetric matrix, the symbol $\star$ stands for the symmetric blocks. $\xi_{t}$ and $\xi_{x}$ stand for the partial derivatives of the function $\xi$ with respect to $t$ and $x$, respectively. For $\xi=\left(\xi_{1}, \ldots, \xi_{n}\right)^{\top} \in C^{0}\left((0, L) ; \mathbb{R}^{n}\right)$, we denote $|\xi|_{0}=\max \left\{|\xi(x)|_{0}, x \in(0, L)\right\}$, with $|\xi(x)|_{0}=\max \left\{\left|\xi_{i}(x)\right|, i=1, \ldots, n\right\} . H^{2}\left((0, L) ; \mathbb{R}^{n}\right)$ is the Sobolev space of all $n$-order $C^{2}$ functions on the open set $(0, L)$, and $\xi \in H^{2}\left((0, L) ; \mathbb{R}^{n}\right)$ means that

$$
\|\xi\|_{H^{2}\left((0, L) ; \mathbb{R}^{n}\right)}^{2}=\int_{0}^{L}\left(|\xi|^{2}+\left|\xi_{x}\right|^{2}+\left|\xi_{x x}\right|^{2}\right) d x<\infty .
$$

2. PI Boundary Control for Hyperbolic Systems. Consider the general quasilinear hyperbolic system of balance laws

$$
\xi_{t}+\Lambda(\xi) \xi_{x}=B(\xi), \quad t \in[0, \infty), \quad x \in(0, L),
$$

where $\xi:[0, L] \times[0, \infty) \rightarrow \mathbb{R}^{n}$ is the vector of the state variables, $\Lambda(\xi) \in \mathbb{R}^{n \times n}$ is the diagonal matrix of $C^{2}$-functions defined as $\Lambda(\xi)=\operatorname{diag}\left\{\Lambda^{+}(\xi),-\Lambda^{-}(\xi)\right\}$, with $\Lambda^{+}(\xi)=\operatorname{diag}\left\{\lambda_{1}(\xi), \ldots, \lambda_{m}(\xi)\right\}$, and $\Lambda^{-}(\xi)=$ $\operatorname{diag}\left\{\lambda_{m+1}(\xi), \ldots, \lambda_{n}(\xi)\right\}, 1 \leq m \leq n$, and eigenvalues $\lambda_{i}(\xi)>0$ for all $i \in\{1, \ldots, n\}, B(\xi) \in C^{2}\left(\mathbb{R}^{n}, \mathbb{R}^{n}\right)$ is the vector function of source terms which satisfies $B(0)=0$ for a given state $\xi=0$.

For simplicity, we use the notations

$$
\xi_{\text {in }}(t):=\left[\begin{array}{c}
\xi^{+}(0, t) \\
\xi^{-}(L, t)
\end{array}\right] \text { and } \xi_{\text {out }}(t):=\left[\begin{array}{c}
\xi^{+}(L, t) \\
\xi^{-}(0, t)
\end{array}\right]
$$

to denote the input and the output of the system (2.1) on the left and the right boundaries, in which the vectors $\xi^{+}$and $\xi^{-}$are defined as $\xi^{+}=\left[\xi_{1}, \ldots, \xi_{m}\right]^{\top}$ and $\xi^{-}=\left[\xi_{m+1}, \ldots, \xi_{n}\right]^{\top}$, respectively.

In this paper, we consider the boundary condition of the PI feedback type, i.e.,

$$
\xi_{\text {in }}(t)=K_{p} \xi_{\text {out }}(t)+K_{i} \int_{0}^{t} \xi_{\text {out }}(\tau) d \tau+\bar{\theta},
$$

where $K_{p} \in \mathbb{R}^{n \times n}, K_{i} \in \mathbb{R}^{n \times n}$ is the proportional and the integral tuning matrices, respectively, and $\bar{\theta} \in \mathbb{R}^{n}$ is an additive outside unknown disturbance which corrupts the system (2.1) on the boundaries of input. In this case, we assume that $\bar{\theta} \in \mathbb{R}^{n}$ is an unknown constant, and $K_{i} \in \mathbb{R}^{n \times n}$ is an invertible matrix. 
The purpose of the paper is to investigate sufficient conditions on the PI boundary feedback controller (2.3), such that the solution of the system 2.1) exists globally, suppresses the static error, and exponentially converges to the state $\xi=0$, for all $x \in[0, L]$, as $t \rightarrow \infty$.

We shall consider the initial condition

$$
\xi(x, 0)=\xi_{o}(x), \quad x \in(0, L)
$$

which satisfies the $C^{0}$ and $C^{1}$ compatibility conditions

$$
\left[\begin{array}{c}
\xi_{o}^{+}(0) \\
\xi_{o}^{-}(L)
\end{array}\right]=K_{p}\left[\begin{array}{c}
\xi_{o}^{+}(L) \\
\xi_{o}^{-}(0)
\end{array}\right]+\bar{\theta}
$$

and

$$
\begin{aligned}
& {\left[\begin{array}{l}
\Lambda^{+}\left(\xi_{o}(0)\right) \partial_{x} \xi_{o}^{+}(0)-B^{+}\left(\xi_{o}(0)\right) \\
-\Lambda^{-}\left(\xi_{o}(L)\right) \partial_{x} \xi_{o}^{-}(L)-B^{-}\left(\xi_{o}(L)\right)
\end{array}\right]} \\
& \quad=K_{p}\left[\begin{array}{c}
\Lambda^{+}\left(\xi_{o}(L)\right) \partial_{x} \xi_{o}^{+}(L)-B^{+}\left(\xi_{o}(L)\right) \\
-\Lambda^{-}\left(\xi_{o}(0)\right) \partial_{x} \xi_{o}^{-}(0)-B^{-}\left(\xi_{o}(0)\right)
\end{array}\right]+K_{i}\left[\begin{array}{c}
\xi_{o}^{+}(L) \\
\xi_{o}^{-}(0)
\end{array}\right]
\end{aligned}
$$

respectively, where the sub maps $B^{+} \in \mathbb{R}^{m}$, and $B^{-} \in \mathbb{R}^{n-m}$ are defined such that $B:=\left[B^{+}, B^{-}\right]^{\top}$.

The well-posedness of the Cauchy problem (2.1), 2.3), 2.4), and the existence of unique classical solutions follows from [4, Appendix B, see precisely Theorem B1 and Corollary B.2] by using the boundary condition (2.3).

TheOREM 2.1. There exists $\delta_{0}>0$ such that, for every $\xi_{o} \in H^{2}\left((0, L) ; \mathbb{R}^{n}\right)$ satisfying $\left\|\xi_{o}\right\|_{H^{2}\left((0, L) ; \mathbb{R}^{n}\right)} \leq \delta_{0}$ and the compatibility conditions (2.5) and (2.6), the Cauchy problem (2.1), (2.3), (2.4) has a unique maximal classical solution

$$
\xi \in C^{0}\left([0, T), H^{2}\left((0, L) ; \mathbb{R}^{n}\right)\right) \cap C^{1}\left([0, T) \times(0, L) ; \mathbb{R}^{n}\right)
$$

with $T \in(0, \infty]$. Moreover, if

$$
\|\xi(\cdot, t)\|_{H^{2}\left((0, L) ; \mathbb{R}^{n}\right)} \leq \delta_{0}, \quad \forall t \in[0, T),
$$

then $T=\infty$.

The definition of the locally exponentially stability for the system $(2.1)$ is given as follows.

DeFinition 2.2. The state $\xi=0$ of the system 2.1), (2.3) is locally exponentially stable (for the $H^{2}$-norm) if there exist $\delta>0, \theta_{\max }(\delta)>0, \nu>0$, and $C>0$ such that, for every $|\bar{\theta}| \leq \theta_{\max }(\delta), \xi_{o} \in H^{2}\left((0, L), \mathbb{R}^{n}\right)$

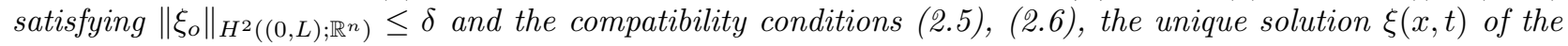
Cauchy problem (2.1), 2.3), 2.4 is defined on $[0, L] \times[0, \infty)$ and satisfies

$$
\|\xi(\cdot, t)\|_{H^{2}\left((0, L) ; \mathbb{R}^{n}\right)} \leq C e^{-\nu t}\left\|\xi_{o}\right\|_{H^{2}\left((0, L) ; \mathbb{R}^{n}\right)}, \quad \forall t \in[0, \infty) .
$$

Define the matrix $M \in \mathbb{R}^{n \times n}$ as the linearization of the source term $B(\xi)$ at the state $\xi=0$, i.e.

$$
M:=\frac{\partial B}{\partial \xi}(0) .
$$

Then the main result of the paper is given as follows.

THEOREM 2.3. If there exist a diagonal matrix $P_{1} \in \mathbb{R}^{n \times n}$, a symmetric matrix $P_{2} \in \mathbb{R}^{n \times n}$, a matrix $P_{3} \in \mathbb{R}^{n \times n}$, and a real constant $\mu \in \mathbb{R}$, satisfying the matrix inequalities, for all $x \in[0, L]$

$$
\begin{aligned}
& \text { (i) } P=\left[\begin{array}{cc}
P_{1} & P_{3} \\
\star & P_{2}
\end{array}\right]>0, \\
& \text { (ii) } \Omega^{e}(x)=\left[\begin{array}{ccccc}
\Omega_{11}^{e}(x) & \Omega_{12}^{e}(x) & P_{3}(x) & 0 & 0 \\
\star & \Omega_{22}^{e} & \Omega_{23}^{e} & 0 & 0 \\
\star & \star & \Omega_{33}^{e} & \Omega_{34}^{e} & 0 \\
\star & \star & \star & \Omega_{44}^{e} & \Omega_{45}^{e} \\
\star & \star & \star & \star & \Omega_{55}^{e}
\end{array}\right]<0,
\end{aligned}
$$


where

$$
\begin{aligned}
& \Omega_{11}^{e}(x)=M^{\top} P_{1}(x)+P_{1}(x) M-2 \mu|\Lambda(0)| P_{1}(x) \\
& \Omega_{12}^{e}(x)=M^{\top} P_{3}(x)-\mu|\Lambda(0)| P_{3}(x) \\
& \Omega_{22}^{e}=\frac{1}{L}\left(K_{i}^{\top}|\Lambda(0)| P_{1} E_{1} K_{i}+K_{i}^{\top}|\Lambda(0)| P_{3} E_{3}+E_{3} P_{3}^{\top}|\Lambda(0)| K_{i}\right), \\
& \Omega_{23}^{e}=\frac{1}{L}\left(K_{i}^{\top}|\Lambda(0)| P_{1} E_{1} K_{p}-|\Lambda(0)| P_{3} E_{4}+E_{3} P_{3}^{\top}|\Lambda(0)| K_{p}\right)+P_{2} \\
& \Omega_{33}^{e}=\frac{1}{L}\left(K_{p}^{\top}|\Lambda(0)| P_{1} E_{1} K_{p}+K_{i}^{\top}|\Lambda(0)| P_{1} E_{1} K_{i}-|\Lambda(0)| P_{1} E_{2}\right) \\
& \Omega_{34}^{e}=\frac{1}{L} K_{i}^{\top}|\Lambda(0)| P_{1} E_{1} K_{p} \\
& \Omega_{44}^{e}=\Omega_{33}^{e} \\
& \Omega_{45}^{e}=\Omega_{34}^{e} \\
& \Omega_{55}^{e}=\frac{1}{L}\left(K_{p}^{\top}|\Lambda(0)| P_{1} E_{1} K_{p}-|\Lambda(0)| P_{1} E_{2}\right)
\end{aligned}
$$

with $P_{1}(x)=P_{1} \operatorname{diag}\left\{e^{-2 \mu x} I_{m}, e^{2 \mu x} I_{n-m}\right\}, P_{3}(x)=P_{3} \operatorname{diag}\left\{e^{-\mu x} I_{m}, e^{\mu x} I_{n-m}\right\},|\Lambda(0)|=\operatorname{diag}\left\{\Lambda^{+}(0), \Lambda^{-}(0)\right\}$, and $E_{1}=\operatorname{diag}\left\{I_{m}, e^{2 \mu L} I_{n-m}\right\}, E_{2}=\operatorname{diag}\left\{e^{-2 \mu L} I_{m}, I_{n-m}\right\}, E_{3}=\sqrt{E_{1}}, E_{4}=\sqrt{E_{2}}$, then the following properties hold true:

- [Local well-posedness] There exist $\delta_{0}>0$ and $\theta_{\max }\left(\delta_{0}\right)>0$ such that, for any unknown constant disturbance $\bar{\theta}$ satisfying $|\bar{\theta}| \leq \theta_{\max }\left(\delta_{0}\right)$, for every $\xi_{o} \in H^{2}\left((0, L), \mathbb{R}^{n}\right)$ satisfying $\left\|\xi_{o}\right\|_{H^{2}\left((0, L) ; \mathbb{R}^{n}\right)} \leq \delta_{0}$ and the compatibility conditions (2.5), (2.6), the Cauchy problem (2.1), (2.3), (2.4) has a classical unique solution $\xi(\cdot, t)$, defined for $t \in[0, \infty)$, such that

$$
\left.\xi(\cdot, t) \in C^{0}\left([0, \infty) ; H^{2}\left((0, L) ; \mathbb{R}^{n}\right)\right) \cap C^{1}\left([0, \infty) \times(0, L) ; \mathbb{R}^{n}\right)\right) .
$$

- [Local exponential stability] The system (2.1) with the PI boundary feedback controller (2.3) is locally exponentially stable for the state $\xi=0$.

Remark 2.4. The static boundary stabilization of the quasilinear hyperbolic system of conservation laws has been solved in [7]. In this paper, as the boundary conditions of the quasilinear hyperbolic system of balance laws (2.1) are corrupted by the unknown constant disturbance, a proportional controller is not suitable, whereas the PI boundary feedback controller (2.3) can be used to suppress the error and hence guarantee the local exponential stabilization of the closed loop system.

Remark 2.5. For the hyperbolic system of balance laws, there is a limitation on the maximal length $L$ above which the stabilization by a static boundary feedback is impossible, as matrices $M=\left[\begin{array}{cc}0 & c \\ c & 0\end{array}\right], K_{p}=\left[\begin{array}{cc}0 & k \\ 1 & 0\end{array}\right]$, $c<0, k \in \mathbb{R}$, (see Section 5 in [3]). In Theorem 2.3. if $L$ is too large, then $\Omega_{55}^{e}>0$, and thus there is no solution to the inequality $\Omega^{e}(x)<0$.

3. Proof of The Main Result. In order to prove Theorem 2.3 , we temporarily assume that the solution $\xi(x, t):[0, L] \times[0, T) \rightarrow \mathbb{R}^{n}$ are of class $C^{3}$, then relax to the condition of class $C^{1}$ later by the density argument developed in [Comment 4.6, page 127] of [4]. The appropriate Lyapunov function candidate $V$ : $H^{2}\left((0, L) ; \mathbb{R}^{n}\right) \times \mathbb{R} \rightarrow \mathbb{R}$ has the following form

$$
V:=V_{1}(\xi, \zeta)+V_{2}\left(\xi_{t}\right)+V_{3}\left(\xi_{t t}\right)
$$

with

$$
\begin{aligned}
V_{1}(\xi, \zeta) & =\int_{0}^{L}\left[\xi^{\top} P_{1}(x) \xi+\xi^{\top} P_{3}(x) \zeta+\zeta^{\top} P_{3}^{\top}(x) \xi\right] d x+L \zeta^{\top} P_{2} \zeta \\
& =\int_{0}^{L}\left[\begin{array}{l}
\xi \\
\zeta
\end{array}\right]^{\top}\left[\begin{array}{cc}
P_{1}(x) & P_{3}(x) \\
\star & P_{2}
\end{array}\right]\left[\begin{array}{l}
\xi \\
\zeta
\end{array}\right] d x \\
V_{2}\left(\xi_{t}\right) & =\int_{0}^{L} \xi_{t}^{\top} P_{1}(x) \xi_{t} d x \\
V_{3}\left(\xi_{t t}\right) & =\int_{0}^{L} \xi_{t t}^{\top} P_{1}(x) \xi_{t t} d x,
\end{aligned}
$$


where $\zeta(t):[0, T) \rightarrow \mathbb{R}$ is the integral of the output of the system 2.1$]$, i.e.,

$$
\zeta(t)=\int_{0}^{t} \xi_{\text {out }}(\tau) d \tau+K_{i}^{-1} \bar{\theta} .
$$

The proof of Theorem 2.3 is based on the estimates of the time derivatives $\dot{V}_{i}, i=1,2,3$, along the solutions of the system (2.1) and (2.3), by expanding the analysis up to the dynamics of $\xi_{t t}$ with the assumption that solutions $\xi$ of class $C^{3}$.

Remark 3.1. The proposed Lyapunov function (3.1) is an extension of the one in [7], in which the static boundary feedback control is considered. When we involve the boundary measurement $\zeta(t)$ of (3.5), the candidate Lyapunov function, more specifically in the first $V_{1}(\xi, \zeta)$ term (3.2), has to be modified to refer to this integral variable.

Remark 3.2. For the quasilinear hyperbolic system of balance laws, it is well known that even for small initial conditions, the shocks may occur in finite time. By means of the Lyapunov function (3.1), the result of Theorem 2.3 guarantees that the shocks will not develop and the classical solutions exist on $t \in[0, \infty)$, for all initial conditions $\left\|\xi_{o}\right\|_{H^{2}\left((0, L) ; \mathbb{R}^{n}\right)} \leq \delta_{0}$ and for any disturbances smaller than the bound $\theta_{\text {max }}\left(\delta_{0}\right)$ (that depends on the size of the initial condition set).

3.1. Proof in the case where $m=n$. We first prove Theorem 2.3 in the special case where $m=n$, which means that the characteristic matrix $\Lambda(\xi)=\Lambda^{+}(\xi)$ with $\lambda_{i}(\xi)>0$, for all $i=1, \ldots, n$.

To simplify the notation, we denote $\xi_{0}(t):=\xi(0, t)$ and $\xi_{L}(t):=\xi(L, t)$. In this case, the PI boundary controller (2.4) is simply rewritten as

$$
\begin{aligned}
\xi_{0}(t) & =K_{p} \xi_{L}(t)+K_{i} \int_{0}^{t} \xi_{L}(\tau) d \tau+\bar{\theta} \\
& =K_{p} \xi_{L}(t)+K_{i} \zeta(t) .
\end{aligned}
$$

Moreover, condition (ii) of Theorem 2.3 is turning into the single matrix inequality as

$$
\left(i i^{\prime}\right) \Omega=\left[\begin{array}{ccccc}
\Omega_{11} & \Omega_{12} & P_{3} & 0 & 0 \\
\star & \Omega_{22} & \Omega_{23} & 0 & 0 \\
\star & \star & \Omega_{33} & \Omega_{34} & 0 \\
\star & \star & \star & \Omega_{44} & \Omega_{45} \\
\star & \star & \star & \star & \Omega_{55}
\end{array}\right]<0
$$

where

$$
\begin{aligned}
& \Omega_{11}=M^{\top} P_{1}+P_{1} M-2 \mu \Lambda(0) P_{1}, \\
& \Omega_{12}=M^{\top} P_{3}-\mu \Lambda(0) P_{3}, \\
& \Omega_{22}=\frac{1}{L}\left(K_{i}^{\top} \Lambda(0) P_{1} K_{i}+K_{i}^{\top} \Lambda(0) P_{3}+P_{3}^{\top} \Lambda(0) K_{i}\right), \\
& \Omega_{23}=\frac{1}{L}\left(K_{i}^{\top} \Lambda(0) P_{1} K_{p}-e^{-\mu L} \Lambda(0) P_{3}+P_{3}^{\top} \Lambda(0) K_{p}\right)+P_{2}, \\
& \Omega_{33}=\frac{1}{L}\left(K_{p}^{\top} \Lambda(0) P_{1} K_{p}+K_{i}^{\top} \Lambda(0) P_{1} K_{i}-e^{-2 \mu L} \Lambda(0) P_{1}\right), \\
& \Omega_{34}=\frac{1}{L} K_{i}^{\top} \Lambda(0) P_{1} K_{p}, \\
& \Omega_{44}=\Omega_{33}, \\
& \Omega_{45}=\Omega_{34}, \\
& \Omega_{55}=\frac{1}{L}\left(K_{p}^{\top} \Lambda(0) P_{1} K_{p}-e^{-2 \mu L} \Lambda(0) P_{1}\right) .
\end{aligned}
$$

Let us firstly introduce a notation to deal with the estimates on the higher order terms. We denote by $\mathcal{O}(X ; Y)$, with $X \geqslant 0$ and $Y \geqslant 0$, quantities for which there exist $C>0$ and $\varepsilon>0$, independent of $\xi, \xi_{t}$ and $\xi_{t t}$, such that

$$
(Y \leqslant \varepsilon) \Rightarrow(|\mathcal{O}(X ; Y)| \leqslant C X) .
$$


Lemma 3.3. The time derivative of $V_{1}(\xi, \zeta)$ along the solutions of 2.1), 2.4), (3.6) is written as follows

$$
\begin{aligned}
\dot{V}_{1}(\xi, \zeta)= & \int_{0}^{L}\left[\begin{array}{c}
\xi e^{-\mu x} \\
\zeta(t) \\
\xi_{L}(t)
\end{array}\right]^{\top}\left[\begin{array}{ccc}
\Omega_{11} & \Omega_{12} & P_{3} \\
\star & \Omega_{22} & \Omega_{23} \\
\star & \star & \Omega_{55}
\end{array}\right]\left[\begin{array}{c}
\xi e^{-\mu x} \\
\zeta(t) \\
\xi_{L}(t)
\end{array}\right] d x \\
& +\mathcal{O}\left(\left|\xi_{L}(t)\right|^{3}+\left|\xi_{L}(t)\right|^{2}|\zeta(t)|+\left|\xi_{L}(t)\right||\zeta(t)|^{2} ;\left|\xi_{L}(t)\right|\right) \\
& +\mathcal{O}\left(\int_{0}^{L}\left(|\xi|^{3}+|\xi|^{2}\left|\xi_{t}\right|+|\xi|^{2}|\zeta(t)|+|\xi|\left|\xi_{t}\right||\zeta(t)|\right) d x ;|\xi|_{0}\right) .
\end{aligned}
$$

Proof: The time derivative of $V_{1}(\xi, \zeta)$ along the solutions of 2.1] is

$$
\begin{aligned}
\dot{V}_{1}(\xi, \zeta)= & \int_{0}^{L}\left[2 \xi_{t}^{\top} P_{1} \xi e^{-2 \mu x}+\xi_{t}^{\top} P_{3} \zeta e^{-\mu x}+\xi^{\top} P_{3} \xi_{L}(t) e^{-\mu x}\right. \\
& \left.+\xi_{L}^{\top}(t) P_{3}^{\top} \xi e^{-\mu x}+\zeta^{\top} P_{3}^{\top} \xi_{t} e^{-\mu x}\right] d x+2 L \zeta^{\top} P_{2} \xi_{L}(t) \\
= & \mathcal{T}_{11}+\mathcal{T}_{12}+\mathcal{T}_{13}
\end{aligned}
$$

in which

$$
\begin{aligned}
& \mathcal{T}_{11}=2 \int_{0}^{L} \xi_{t}^{\top} P_{1} \xi e^{-2 \mu x} d x, \\
& \mathcal{T}_{12}=\int_{0}^{L}\left[\xi_{t}^{\top} P_{3} \zeta+\xi^{\top} P_{3} \xi_{L}(t)+\xi_{L}^{\top}(t) P_{3}^{\top} \xi+\zeta^{\top} P_{3}^{\top} \xi_{t}\right] e^{-\mu x} d x, \\
& \mathcal{T}_{13}=2 L \zeta^{\top} P_{2} \xi_{L}(t) .
\end{aligned}
$$

Using the nonlinear equation $(2.1)$, and performing an integration by parts for the first two terms $\mathcal{T}_{11}$ and $\mathcal{T}_{12}$, respectively, we get

$$
\begin{aligned}
\mathcal{T}_{11}= & 2 \int_{0}^{L}\left[-\Lambda(\xi) \xi_{x}+B(\xi)\right]^{\top} P_{1} \xi e^{-2 \mu x} d x \\
= & {\left[-\xi^{\top} \Lambda(\xi) P_{1} \xi e^{-2 \mu x}\right]_{0}^{L} } \\
& +\int_{0}^{L} \xi^{\top} \frac{\partial \Lambda}{\partial \xi}(\xi) \xi_{x} P_{1} \xi e^{-2 \mu x} d x \\
& +\int_{0}^{L}\left[-2 \mu \xi^{\top} \Lambda(\xi) P_{1} \xi+B^{\top}(\xi) P_{1} \xi+\xi^{\top} P_{1} B(\xi)\right] e^{-2 \mu x} d x
\end{aligned}
$$

and

$$
\begin{aligned}
\mathcal{T}_{12}= & \int_{0}^{L}\left[-\Lambda(\xi) \xi_{x}+B(\xi)\right]^{\top} P_{3} \zeta e^{-\mu x} d x \\
& +\int_{0}^{L} \zeta^{\top} P_{3}^{\top}\left[-\Lambda(\xi) \xi_{x}+B(\xi)\right] e^{-\mu x} d x \\
& +\int_{0}^{L}\left[\xi^{\top} P_{3} \xi_{L}(t)+\xi_{L}^{\top}(t) P_{3}^{\top} \xi\right] e^{-\mu x} d x \\
= & -\left[\xi^{\top} \Lambda(\xi) P_{3} \zeta e^{-\mu x}+\zeta^{\top} P_{3}^{\top} \Lambda(\xi) \xi e^{-\mu x}\right]_{0}^{L} \\
& -\mu \int_{0}^{L}\left[\xi^{\top} \Lambda(\xi) P_{3} \zeta+\zeta^{\top} P_{3}^{\top} \Lambda(\xi) \xi\right] e^{-\mu x} d x \\
& +\int_{0}^{L}\left[\xi^{\top} \frac{\partial \Lambda}{\partial \xi}(\xi) \xi_{x} P_{3} \zeta+\zeta^{\top} P_{3}^{\top} \frac{\partial \Lambda}{\partial \xi}(\xi) \xi_{x} \xi\right] e^{-\mu x} d x \\
& +\int_{0}^{L}\left[B^{\top}(\xi) P_{3} \zeta+\zeta^{\top} P_{3}^{\top} B(\xi)+\xi^{\top} P_{3} \xi_{L}(t)+\xi_{L}^{\top}(t) P_{3}^{\top} \xi\right] e^{-\mu x} d x .
\end{aligned}
$$


Then, combine the feedback gain matrices $K_{p}$, and $K_{i}$ of the PI boundary controller (3.6), and the linearized matrix $M$ of the source term $(2.10)$ into equations $(3.13)$ and $(3.14)$, respectively, we have

$$
\begin{aligned}
& \mathcal{T}_{11}=-\xi_{L}^{\top}(t) \Lambda(0) P_{1} \xi_{L}(t) e^{-2 \mu L}+\left[K_{p} \xi_{L}(t)+K_{i} \zeta\right]^{\top} \Lambda(0) P_{1}\left[K_{p} \xi_{L}(t)+K_{i} \zeta\right] \\
& +\mathcal{O}\left(\left|\xi_{L}(t)\right|^{3}+\left|\xi_{L}(t)\right|^{2}|\zeta(t)|+|\zeta(t)|^{2}\left|\xi_{L}(t)\right| ;\left|\xi_{L}(t)\right|\right) \\
& +\int_{0}^{L}\left[-2 \mu \xi^{\top} \Lambda(0) P_{1} \xi+\xi^{\top} M^{\top} P_{1} \xi+\xi^{\top} P_{1} M \xi\right] e^{-2 \mu x} d x \\
& +\mathcal{O}\left(\int_{0}^{L}\left(|\xi|^{3}+|\xi|^{2}\left|\xi_{t}\right|\right) d x ;|\xi|_{0}\right) \\
& =\int_{0}^{L} \xi^{\top}\left[M^{\top} P_{1}+P_{1} M-2 \mu \Lambda(0) P_{1}\right] \xi e^{-2 \mu x} d x \\
& +\left[\begin{array}{c}
\zeta(t) \\
\xi_{L}(t)
\end{array}\right]^{\top}\left[\begin{array}{cc}
K_{i}^{\top} \Lambda(0) P_{1} K_{i} & K_{i}^{\top} \Lambda(0) P_{1} K_{p} \\
\star & K_{p}^{\top} \Lambda(0) P_{1} K_{p}-e^{-2 \mu L} \Lambda(0) P_{1}
\end{array}\right]\left[\begin{array}{c}
\zeta(t) \\
\xi_{L}(t)
\end{array}\right] \\
& +\mathcal{O}\left(\left|\xi_{L}(t)\right|^{3}+\left|\xi_{L}(t)\right|^{2}|\zeta(t)|+|\zeta(t)|^{2}\left|\xi_{L}(t)\right| ;\left|\xi_{L}(t)\right|\right) \\
& +\mathcal{O}\left(\int_{0}^{L}\left(|\xi|^{3}+|\xi|^{2}\left|\xi_{t}\right|\right) d x ;|\xi|_{0}\right) \\
& =\int_{0}^{L}\left[\begin{array}{c}
\xi e^{-\mu x} \\
\zeta(t) \\
\xi_{L}(t)
\end{array}\right]^{\top}\left[\begin{array}{ccc}
\Omega_{11} & 0 & 0 \\
\star & \frac{K_{i}^{\top} \Lambda(0) P_{1} K_{i}}{L} & \frac{K_{i}^{\top} \Lambda(0) P_{1} K_{p}}{L} \\
\star & \star & \Omega_{55}
\end{array}\right]\left[\begin{array}{c}
\xi e^{-\mu x} \\
\zeta(t) \\
\xi_{L}(t)
\end{array}\right] \\
& +\mathcal{O}\left(\left|\xi_{L}(t)\right|^{3}+\left|\xi_{L}(t)\right|^{2}|\zeta(t)|+|\zeta(t)|^{2}\left|\xi_{L}(t)\right| ;\left|\xi_{L}(t)\right|\right) \\
& +\mathcal{O}\left(\int_{0}^{L}\left(|\xi|^{3}+|\xi|^{2}\left|\xi_{t}\right|\right) d x ;|\xi|_{0}\right)
\end{aligned}
$$

and

$$
\begin{aligned}
& \mathcal{T}_{12}=-\xi_{L}^{\top}(t) \Lambda(0) P_{3} \zeta e^{-\mu L}-\zeta^{\top} P_{3}^{\top} \Lambda(0) \xi_{L}(t) e^{-\mu L} \\
& +\left[K_{p} \xi_{L}(t)+K_{i} \zeta\right]^{\top} \Lambda(0) P_{3} \zeta+\zeta^{\top} P_{3}^{\top} \Lambda(0)\left[K_{p} \xi_{L}(t)+K_{i} \zeta\right] \\
& +\mathcal{O}\left(\left|\xi_{L}(t)\right|^{2}|\zeta(t)|+|\zeta(t)|^{2}\left|\xi_{L}(t)\right| ;\left|\xi_{L}(t)\right|\right) \\
& -\mu \int_{0}^{L}\left[\xi^{\top} \Lambda(0) P_{3} \zeta+\zeta^{\top} P_{3}^{\top} \Lambda(0) \xi\right] e^{-\mu x} d x \\
& +\int_{0}^{L}\left[\xi^{\top} M^{\top} P_{3} \zeta+\zeta^{\top} P_{3}^{\top} M \xi+\xi^{\top} P_{3} \xi_{L}(t)+\xi_{L}^{\top}(t) P_{3}^{\top} \xi\right] e^{-\mu x} d x \\
& +\mathcal{O}\left(\int_{0}^{L}\left(|\xi|^{2}|\zeta(t)|+|\xi|\left|\xi_{t}\right||\zeta(t)|\right) d x ;|\xi|_{0}\right) \\
& =\left[\begin{array}{c}
\zeta(t) \\
\xi_{L}(t)
\end{array}\right]^{\top}\left[\begin{array}{cc}
K_{i}^{\top} \Lambda(0) P_{3}+P_{3}^{\top} \Lambda(0) K_{i} & P_{3}^{\top} \Lambda(0) K_{p}-e^{-\mu L} \Lambda(0) P_{3} \\
\star & 0
\end{array}\right]\left[\begin{array}{c}
\zeta(t) \\
\xi_{L}(t)
\end{array}\right] \\
& +\int_{0}^{L}\left[\begin{array}{c}
\xi e^{-\mu x} \\
\zeta(t) \\
\xi_{L}(t)
\end{array}\right]^{\top}\left[\begin{array}{ccc}
0 & M^{\top} P_{3}-\mu \Lambda(0) P_{3} & P_{3} \\
\star & 0 & 0 \\
\star & \star & 0
\end{array}\right]\left[\begin{array}{c}
\xi e^{-\mu x} \\
\zeta(t) \\
\xi_{L}(t)
\end{array}\right] d x \\
& +\mathcal{O}\left(\left|\xi_{L}(t)\right|^{2}|\zeta(t)|+|\zeta(t)|^{2}\left|\xi_{L}(t)\right| ;\left|\xi_{L}(t)\right|\right) \\
& +\mathcal{O}\left(\int_{0}^{L}\left(|\xi|^{2}|\zeta(t)|+|\xi|\left|\xi_{t}\right||\zeta(t)|\right) d x ;|\xi|_{0}\right)
\end{aligned}
$$




$$
\begin{aligned}
= & \int_{0}^{L}\left[\begin{array}{c}
\xi e^{-\mu x} \\
\zeta(t) \\
\xi_{L}(t)
\end{array}\right]^{\top}\left[\begin{array}{ccc}
0 & \Omega_{12} & P_{3} \\
\star & \frac{K_{i}^{\top} \Lambda(0) P_{3}+P_{3}^{\top} \Lambda(0) K_{i}}{L} & \frac{P_{3}^{\top} \Lambda(0) K_{p}-e^{-\mu L} \Lambda(0) P_{3}}{L} \\
\star & \star & 0
\end{array}\right]\left[\begin{array}{c}
\xi e^{-\mu x} \\
\zeta(t) \\
\xi_{L}(t)
\end{array}\right] d x \\
& +\mathcal{O}\left(\left|\xi_{L}(t)\right|^{2}|\zeta(t)|+|\zeta(t)|^{2}\left|\xi_{L}(t)\right| ;\left|\xi_{L}(t)\right|\right) \\
& +\mathcal{O}\left(\int_{0}^{L}\left(|\xi|^{2}|\zeta(t)|+|\xi|\left|\xi_{t}\right||\zeta(t)|\right) d x ;|\xi|_{0}\right) .
\end{aligned}
$$

Moreover, the term $\mathcal{T}_{13}$ in 3.12 may be rewritten as

$$
\mathcal{T}_{13}=L\left[\begin{array}{c}
\zeta(t) \\
\xi_{L}(t)
\end{array}\right]^{\top}\left[\begin{array}{cc}
0 & P_{2} \\
\star & 0
\end{array}\right]\left[\begin{array}{c}
\zeta(t) \\
\xi_{L}(t)
\end{array}\right] .
$$

Substituting the block matrices of $\mathcal{T}_{13}$ in (3.17) into the integral term of $\mathcal{T}_{12}$ in (3.16), and further combining $\mathcal{T}_{11}$ in 3.15 , we finally get the estimate

$$
\begin{aligned}
\dot{V}_{1}(\xi, \zeta)= & \int_{0}^{L}\left[\begin{array}{c}
\xi e^{-\mu x} \\
\zeta(t) \\
\xi_{L}(t)
\end{array}\right]^{\top}\left[\begin{array}{ccc}
\Omega_{11} & \Omega_{12} & P_{3} \\
\star & \Omega_{22} & \Omega_{23} \\
\star & \star & \Omega_{55}
\end{array}\right]\left[\begin{array}{c}
\xi e^{-\mu x} \\
\zeta(t) \\
\xi_{L}(t)
\end{array}\right] d x \\
& +\mathcal{O}\left(\left|\xi_{L}(t)\right|^{3}+\left|\xi_{L}(t)\right|^{2}|\zeta(t)|+\left|\xi_{L}(t)\right||\zeta(t)|^{2} ;\left|\xi_{L}(t)\right|\right) \\
& +\mathcal{O}\left(\int_{0}^{L}\left(|\xi|^{3}+|\xi|^{2}\left|\xi_{t}\right|+|\xi|^{2}|\zeta(t)|+|\xi|\left|\xi_{t}\right||\zeta(t)|\right) d x ;|\xi|_{0}\right) .
\end{aligned}
$$

This concludes the proof of Lemma 3.3

Under the assumption that the solution $\xi$ of class $C^{3}$, maps $\Lambda, B$ are of class $C^{3}$, by the time differentiation of (2.1) and (2.3), and by recalling the integral of $(3.5), \xi_{t}:[0, L] \times[0, T) \rightarrow \mathbb{R}^{n}$ satisfies the following hyperbolic dynamics:

$$
\xi_{t t}+\Lambda(\xi) \xi_{t x}+\operatorname{diag}\left[\frac{\partial \Lambda}{\partial \xi}(\xi) \xi_{t}\right] \xi_{x}=\frac{\partial B}{\partial \xi}(\xi) \xi_{t}
$$

with the boundary condition

$$
\xi_{t}(0, t)=K_{p} \xi_{t}(L, t)+K_{i} \xi_{L}(t)
$$

where, the matrix $\operatorname{diag}\left[(\partial \Lambda / \partial \xi) \xi_{t}\right]$ stands for the diagonal matrix whose diagonal entries are the components of the vector $(\partial \Lambda / \partial \xi) \xi_{t}$.

LEMma 3.4. The time derivative of $V_{2}\left(\xi_{t}\right)$ along the solutions of (3.19), (3.20) is written as follows

$$
\begin{aligned}
\dot{V}_{2}\left(\xi_{t}\right)= & \int_{0}^{L} \xi_{t}^{\top} \Omega_{11} \xi_{t} e^{-2 \mu x} d x \\
& +\left[\begin{array}{c}
\xi_{L}(t) \\
\xi_{t}(L, t)
\end{array}\right]^{\top}\left[\begin{array}{cc}
K_{i}^{\top} \Lambda(0) P_{1} K_{i} & K_{i}^{\top} \Lambda(0) P_{1} K_{p} \\
\star & K_{p}^{\top} \Lambda(0) P_{1} K_{p}-e^{-2 \mu L} \Lambda(0) P_{1}
\end{array}\right]\left[\begin{array}{c}
\xi_{L}(t) \\
\xi_{t}(L, t)
\end{array}\right] \\
& +\mathcal{O}\left(\left|\xi_{t}(L, t)\right|^{2}\left|\xi_{L}(t)\right|+\left|\xi_{L}(t)\right|^{2}\left|\xi_{t}(L, t)\right|+\left|\xi_{L}(t)\right|^{3} ;\left|\xi_{L}(t)\right|\right) \\
& +\mathcal{O}\left(\int_{0}^{L}\left(\left|\xi_{t}\right|^{2}|\xi|+\left|\xi_{t}\right|^{3}\right) d x ;|\xi|_{0}\right)
\end{aligned}
$$

The proof of this result is given in Appendix A.

Since $\Lambda, B, \xi$ are of class $C^{3}$, by time differentiation of the system equations 3.19 and $3.20, \xi_{t t}$ : $[0, L] \times[0, T) \rightarrow \mathbb{R}^{n}$ can be shown to satisfy the following hyperbolic dynamics:

$$
\xi_{t t t}+\Lambda(\xi) \xi_{t t x}+2 \operatorname{diag}\left[\frac{\partial \Lambda}{\partial \xi}(\xi) \xi_{t}\right] \xi_{t x}+\operatorname{diag}\left[\frac{\partial \Lambda}{\partial \xi}(\xi) \xi_{t}\right]_{t} \xi_{x}=\frac{\partial B}{\partial \xi}(\xi) \xi_{t t}+\left[\frac{\partial B}{\partial \xi}(\xi)\right]_{t} \xi_{t}
$$


with the boundary condition

$$
\xi_{t t}(0, t)=K_{p} \xi_{t t}(L, t)+K_{i} \xi_{t}(L, t) .
$$

Lemma 3.5. The time derivative of $V_{3}\left(\xi_{t t}\right)$ along the solutions of (3.22), 3.23) is written as follows

$$
\begin{aligned}
\dot{V}_{3}\left(\xi_{t t}\right)= & \int_{0}^{L} \xi_{t t}^{\top} \Omega_{11} \xi_{t t} e^{-2 \mu x} d x \\
& +\left[\begin{array}{c}
\xi_{t}(L, t) \\
\xi_{t t}(L, t)
\end{array}\right]^{\top}\left[\begin{array}{cc}
K_{i}^{\top} \Lambda(0) P_{1} K_{i} & K_{i}^{\top} \Lambda(0) P_{1} K_{p} \\
\star & K_{p}^{\top} \Lambda(0) P_{1} K_{p}-e^{-2 \mu L} \Lambda(0) P_{1}
\end{array}\right]\left[\begin{array}{c}
\xi_{t}(L, t) \\
\xi_{t t}(L, t)
\end{array}\right] \\
& +\mathcal{O}\left(\left|\xi_{t t}(L, t)\right|^{2}\left|\xi_{L}(t)\right|+\left|\xi_{t}(L, t)\right|^{2}\left|\xi_{L}(t)\right|+\left|\xi_{t t}(L, t)\right|\left|\xi_{t}(L, t)\right|\left|\xi_{L}(t)\right| ;\left|\xi_{L}(t)\right|\right) \\
& +\mathcal{O}\left(\int_{0}^{L}\left(\left|\xi_{t t}\right|^{2}|\xi|+\left|\xi_{t t}\right|^{2}\left|\xi_{t}\right|+\left|\xi_{t t}\right|\left|\xi_{t}\right|^{2}\right) d x ;|\xi|_{0}+\left|\xi_{t}\right|_{0}\right)
\end{aligned}
$$

The proof of this result is given in Appendix B.

LEMma 3.6. There exist constants $\alpha>0, \beta>0$, and $\delta>0$, such that for every solution $\xi:(0, L) \times[0, T) \rightarrow$ $\mathbb{R}^{n}$ of (2.1) and (3.6), satisfying $|\xi|_{0}+\left|\xi_{t}\right|_{0} \leq \delta$, we have

$$
\begin{aligned}
\frac{1}{\beta}\left(\int_{0}^{L}\left(|\xi|^{2}+\left|\xi_{x}\right|^{2}+\left|\xi_{x x}\right|^{2}\right) d x+|\zeta(t)|^{2}\right) & \leq V \leq \beta\left(\int_{0}^{L}\left(|\xi|^{2}+\left|\xi_{x}\right|^{2}+\left|\xi_{x x}\right|^{2}\right) d x+|\zeta(t)|^{2}\right), \\
\dot{V} & \leq-\alpha V .
\end{aligned}
$$

Proof: From the system equations (2.1) and (3.19), we know that

$$
\begin{gathered}
\xi_{t}=-\Lambda(\xi) \xi_{x}+B(\xi), \\
\xi_{t t}=\Lambda(\xi)\left[\Lambda(\xi) \xi_{x}-B(\xi)\right]_{x}+\operatorname{diag}\left[\frac{\partial \Lambda}{\partial \xi}(\xi)\left(\Lambda(\xi) \xi_{x}-B(\xi)\right)\right] \xi_{x}-\frac{\partial B}{\partial \xi}(\xi)\left(\Lambda(\xi) \xi_{x}-B(\xi)\right) .
\end{gathered}
$$

Using the expressions (3.27), (3.28), inequality (3.25) follows directly from the definition of Lyapunov function $V$ and the straightforward estimates as $\beta$ is large enough.

Let us introduce the compact vector notation:

$$
\phi^{\top}(x, t):=\left[\xi e^{-\mu x}, \zeta(t), \xi_{L}(t), \xi_{t}(L, t), \xi_{t t}(L, t)\right]^{\top} .
$$

Then, for every solution $\xi:(0, L) \times[0, T) \rightarrow \mathbb{R}^{n}$ of $(2.1)$ and $(3.6)$, it follows from Lemmas 3.3, 3.4, and 3.5 that

$$
\begin{aligned}
\dot{V}= & \dot{V}_{1}(\xi, \zeta)+\dot{V}_{2}\left(\xi_{t}\right)+\dot{V}_{3}\left(\xi_{t t}\right) \\
= & \int_{0}^{L} \phi^{\top}(x, t) \Omega \phi(x, t) d x+\int_{0}^{L} \xi_{t}^{\top} \Omega_{11} \xi_{t} e^{-2 \mu x} d x+\int_{0}^{L} \xi_{t t}^{\top} \Omega_{11} \xi_{t t} e^{-2 \mu x} d x \\
& +\mathcal{O}\left(\left|\xi_{L}(t)\right|\left(|\zeta(t)|^{2}+\left|\xi_{L}(t)\right|^{2}+\left|\xi_{t}(L, t)\right|^{2}+\left|\xi_{t t}(L, t)\right|^{2}+|\zeta(t)|\left|\xi_{L}(t)\right|\right)\right. \\
& \left.\quad+\left|\xi_{t}(L, t)\right|\left|\xi_{L}(t)\right|^{2}+\left|\xi_{L}(t)\right|\left|\xi_{t}(L, t)\right| \xi_{t t}(L, t)|;| \xi_{L}(t) \mid\right) . \\
& \left.+\mathcal{O}\left(\int_{0}^{L}\left(|\xi|+\left|\xi_{t}\right|\right)\left(|\xi|^{2}+\left|\xi_{t}\right|^{2}+\left|\xi_{t t}\right|^{2}\right)+|\zeta(t)||\xi|^{2}+\left|\xi_{t t}\right|\left|\xi_{t}\right|^{2}+|\zeta(t)||\xi|\left|\xi_{t}\right|\right) d x ;|\xi|_{0}+\left|\xi_{t}\right|_{0}\right) .
\end{aligned}
$$

Since $\Omega<0$, it follows that $\phi^{\top}(x, t) \Omega \phi(x, t)$ is a strictly negative definite quadratic form in $\zeta(t), \xi_{L}(t), \xi_{t}(L, t)$, and $\xi_{t t}(L, t)$. Consequently, there exist a $\delta_{1}>0$ and $\alpha_{1}>0$, such that, if $\left|\xi_{L}(t)\right| \leq \delta_{1}$, then

$$
\begin{aligned}
\dot{V} \leq & -\alpha_{1} \int_{0}^{L}|\phi(x, t)|^{2} d x+\int_{0}^{L} \xi_{t}^{\top} \Omega_{11} \xi_{t} e^{-2 \mu x} d x+\int_{0}^{L} \xi_{t t}^{\top} \Omega_{11} \xi_{t t} e^{-2 \mu x} d x \\
& \left.+\mathcal{O}\left(\int_{0}^{L}\left(|\xi|+\left|\xi_{t}\right|\right)\left(|\xi|^{2}+\left|\xi_{t}\right|^{2}+\left|\xi_{t t}\right|^{2}\right)+|\zeta(t)||\xi|^{2}+\left|\xi_{t t}\right|\left|\xi_{t}\right|^{2}+|\zeta(t)||\xi|\left|\xi_{t}\right|\right) d x ;|\xi|_{0}+\left|\xi_{t}\right|_{0}\right) .
\end{aligned}
$$


For every $\eta>0$, we have

$$
\begin{aligned}
\int_{0}^{L}|\zeta(t)||\xi|^{2} d x & \leq \int_{0}^{L}\left(\frac{1}{4 \eta}|\xi|^{4}+\eta|\zeta(t)|^{2}\right) d x \\
& \leq \frac{1}{4 \eta}|\xi|_{0}^{2} \int_{0}^{L}|\xi|^{2} d x+\eta \int_{0}^{L}|\zeta(t)|^{2} d x \\
\int_{0}^{L}\left|\xi_{t t}\right|\left|\xi_{t}\right|^{2} d x & \leq \frac{1}{4 \eta}\left|\xi_{t}\right|_{0}^{2} \int_{0}^{L}\left|\xi_{t}\right|^{2} d x+\eta \int_{0}^{L}\left|\xi_{t t}\right|^{2} d x \\
\int_{0}^{L}|\zeta(t)||\xi|\left|\xi_{t}\right| d x & \leq\left|\xi_{t}\right|_{0} \int_{0}^{L}|\zeta(t)||\xi| d x \\
& \leq \frac{1}{4 \eta}\left|\xi_{t}\right|_{0} \int_{0}^{L}|\xi|^{2} d x+\eta\left|\xi_{t}\right|_{0} \int_{0}^{L}|\zeta(t)|^{2} d x
\end{aligned}
$$

By combing the inequalities (3.32), (3.33), and (3.34), it follows that exist $\delta_{2}>0$ and $C>0$, such that, if $|\xi|_{0}+\left|\xi_{t}\right|_{0} \leq \delta_{2}$, then

$$
\begin{aligned}
& \left.\mathcal{O}\left(\int_{0}^{L}\left(|\xi|+\left|\xi_{t}\right|\right)\left(|\xi|^{2}+\left|\xi_{t}\right|^{2}+\left|\xi_{t t}\right|^{2}\right)+|\zeta(t)||\xi|^{2}+\left|\xi_{t t}\right|\left|\xi_{t}\right|^{2}+|\zeta(t)||\xi|\left|\xi_{t}\right|\right) d x ;|\xi|_{0}+\left|\xi_{t}\right|_{0}\right) \\
& \leq C\left(|\xi|_{0}+\left|\xi_{t}\right|_{0}\right) V .
\end{aligned}
$$

Moreover, using $\Omega_{11}<0$ as $\Omega<0$, there exist $\alpha_{2}>0$ such that

$$
-\alpha_{1} \int_{0}^{L}|\phi(x, t)|^{2} d x+\int_{0}^{L} \xi_{t}^{\top} \Omega_{11} \xi_{t} e^{-2 \mu x} d x+\int_{0}^{L} \xi_{t t}^{\top} \Omega_{11} \xi_{t t} e^{-2 \mu x} d x \leq-\alpha_{2} V .
$$

It follows from 3.30 that, if $\delta<\min \left\{\delta_{1}, \delta_{2}\right\}$ is taken sufficiently small, then $\alpha>0$ can be selected such that

$$
\dot{V} \leq\left(-\alpha_{2}+C\left(|\xi|_{0}+\left|\xi_{t}\right|_{0}\right)\right) V \leq-\alpha V,
$$

for every $\xi$ with $|\xi|_{0}+\left|\xi_{t}\right|_{0} \leq \delta$. This concludes the proof of Lemma 3.6

3.2. Proof in the case where $0<m<n$. In this section, we explain the modifications of the proof of Lemma 3.6 that used to deal with the case $0<m<n$. The case $m=0$ is equivalent to the case $m=n$ by considering $\xi(L-x, t)$ instead of $\xi(x, t)$.

The major difference lies in the functionals $\dot{V}_{1}(\xi, \zeta), \dot{V}_{2}\left(\xi_{t}\right)$ and $\dot{V}_{3}\left(\xi_{t t}\right)$ which are now rewritten, respectively, as follows:

$$
\begin{aligned}
\dot{V}_{1}(\xi, \zeta)= & \int_{0}^{L}\left[\begin{array}{c}
\xi \\
\zeta(t) \\
\xi_{\text {out }}(t)
\end{array}\right]^{\top}\left[\begin{array}{ccc}
\Omega_{11}^{e}(x) & \Omega_{12}^{e}(x) & P_{3}(x) \\
\star & \Omega_{22}^{e} & \Omega_{23}^{e} \\
\star & \star & \Omega_{55}^{e}
\end{array}\right]\left[\begin{array}{c}
\xi \\
\zeta(t) \\
\xi_{\text {out }}(t)
\end{array}\right] d x \\
& +\mathcal{O}\left(\left|\xi_{\text {out }}(t)\right|^{3}+\left|\xi_{\text {out }}(t)\right|^{2}|\zeta(t)|+\left|\xi_{\text {out }}(t)\right||\zeta(t)|^{2} ;\left|\xi_{\text {out }}(t)\right|\right) \\
& +\mathcal{O}\left(\int_{0}^{L}\left(|\xi|^{3}+|\xi|^{2}\left|\xi_{t}\right|+|\xi|^{2}|\zeta(t)|+|\xi|\left|\xi_{t}\right||\zeta(t)|\right) d x ;|\xi| 0\right), \\
\dot{V}_{2}\left(\xi_{t}\right)= & \int_{0}^{L} \xi_{t}^{\top} \Omega_{11}^{e}(x) \xi_{t} d x \\
& +\left[\begin{array}{l}
\xi_{\text {out }}(t) \\
\xi_{\text {out }}^{\prime}(t)
\end{array}\right]^{\top}\left[\begin{array}{cc}
K_{i}^{\top}|\Lambda(0)| P_{1} E_{1} K_{i} & K_{i}^{\top}|\Lambda(0)| P_{1} E_{1} K_{p} \\
\star & L \Omega_{55}^{e}
\end{array}\right]\left[\begin{array}{c}
\xi_{\text {out }}(t) \\
\xi_{\text {out }}^{\prime}(t)
\end{array}\right] \\
& +\mathcal{O}\left(\left|\xi_{\text {out }}^{\prime}(t)\right|^{2}\left|\xi_{\text {out }}(t)\right|+\left|\xi_{\text {out }}(t)\right|^{2}\left|\xi_{\text {out }}^{\prime}(t)\right|+\left|\xi_{\text {out }}(t)\right|^{3} ;\left|\xi_{\text {out }}(t)\right|\right) \\
& +\mathcal{O}\left(\int_{0}^{L}\left(\left|\xi_{t}\right|^{3}+\left|\xi_{t}\right|^{2}|\xi|\right) d x ;|\xi|_{0}\right),
\end{aligned}
$$




$$
\begin{aligned}
\dot{V}_{3}\left(\xi_{t t}\right)= & \int_{0}^{L} \xi_{t t}^{\top} \Omega_{11}^{e}(x) \xi_{t t} d x \\
& +\left[\begin{array}{c}
\xi_{\text {out }}^{\prime}(t) \\
\xi_{\text {out }}^{\prime \prime}(t)
\end{array}\right]^{\top}\left[\begin{array}{cc}
K_{i}^{\top}|\Lambda(0)| P_{1} E_{1} K_{i} & K_{i}^{\top}|\Lambda(0)| P_{1} E_{1} K_{p} \\
\star & L \Omega_{55}^{e}
\end{array}\right]\left[\begin{array}{c}
\xi_{\text {out }}^{\prime}(t) \\
\xi_{\text {out }}^{\prime \prime}(t)
\end{array}\right] \\
& +\mathcal{O}\left(\left|\xi_{\text {out }}^{\prime \prime}(t)\right|^{2}\left|\xi_{\text {out }}(t)\right|+\left|\xi_{\text {out }}^{\prime}(t)\right|^{2}\left|\xi_{\text {out }}(t)\right|+\left|\xi_{\text {out }}^{\prime \prime}(t)\right|\left|\xi_{\text {out }}^{\prime}(t)\right|\left|\xi_{\text {out }}(t)\right| ;\left|\xi_{\text {out }}(t)\right|\right) \\
& +\mathcal{O}\left(\int_{0}^{L}\left(\left|\xi_{t t}\right|^{2}\left|\xi_{t}\right|+\left|\xi_{t t}\right|^{2}|\xi|+\left|\xi_{t t}\right|\left|\xi_{t}\right|^{2}\right) d x ;|\xi|_{0}+\left|\xi_{t}\right|_{0}\right) .
\end{aligned}
$$

Under the conditions (i) and (ii) that matrices $P>0$ and $\Omega^{e}(x)<0$, it is straightforward to verify that Lemma 3.6 can be established for the case $0<m<n$ in a manner completely parallel to the one that we have followed in the case $m=n$.

3.3. Proof of Theorem 2.3. The temporary assumption that $\xi$ of class $C^{3}$ is relaxed in this subsection. In Lemma 3.6, although the estimates $(3.25)$ and $(3.26)$ are obtained under the assumption that maps $\Lambda$ and $B$ are of class $C^{3}$, and the solution $\xi$ of class $C^{3}$, the selection of $\alpha, \beta$ depends only on the $C^{2}$-norm of $\Lambda, B$ and the $C^{0}\left([0, T] ; H^{2}\left((0, L) ; \mathbb{R}^{n}\right)\right)$-norm of $\xi$, (see, e.g., [4, Comment 4.6, page 127]). Hence, using the classical density argument, the estimates 3.25 and 3.26 remain valid in the distribution sense with $\Lambda, B$ of class $C^{2}$, and $\xi$ is only of class $C^{1}$.

For every $\varphi$ in the Sobolev space $H^{2}\left((0, L) ; \mathbb{R}^{n}\right)$, by Sobolev inequality (see e.g., [25]), there exists $C_{1}>0$ such that

$$
|\varphi|_{0} \leq C_{1}\left(\int_{0}^{L}\left(|\varphi|^{2}+\left|\varphi_{x}\right|^{2}\right) d x\right)^{\frac{1}{2}} .
$$

In order to take the time derivative instead of the space derivative and use Lemma 3.6, we directly recall the results in [7, Appendix Lemma B.6, B.7] that there exists $C_{2}>0$ such that, if $|\varphi|_{0}<\delta_{0}$, then

$$
\begin{gathered}
\left|\varphi_{t}\right| \leq C_{2}\left(|\varphi|+\left|\varphi_{x}\right|\right), \\
\left|\varphi_{x t}\right| \leq C_{2}\left(|\varphi|+\left|\varphi_{x}\right|+\left|\varphi_{x x}\right|\right) .
\end{gathered}
$$

Therefore, there exists $C_{0}>0$ such that

$$
|\varphi|_{0}+\left|\varphi_{t}\right|_{0} \leq C_{0}\|\varphi\|_{H^{2}\left((0, L) ; \mathbb{R}^{n}\right)}
$$

for all $|\varphi|_{0}<\delta_{0}$. Let

$$
\varepsilon \triangleq \min \left\{\frac{\delta}{2 C_{0} \beta}, \frac{\delta_{0}}{\beta}\right\}
$$

where $\beta \geq 1$ and therefore that $\varepsilon \leq \delta_{0}$. Using Lemma 3.6 (3.44) and 3.45, for every $t \in[0, T]$ :

$$
\begin{aligned}
\|\xi(\cdot, t)\|_{H^{2}\left((0, L) ; \mathbb{R}^{n}\right)} & \leq \varepsilon \\
\Longrightarrow|\xi(\cdot, t)|_{0}+\left|\xi_{t}(\cdot, t)\right|_{0} & \leq \frac{\delta}{2} \text { and } V \leq \beta \varepsilon^{2}+\beta \zeta^{2}(t), \\
\Longrightarrow\|\xi(\cdot, t)\|_{H^{2}\left((0, L) ; \mathbb{R}^{n}\right)} & \leq \delta_{0} \text { and } \dot{V} \leq 0 .
\end{aligned}
$$

Let $\xi_{o} \in H^{2}\left((0, L) ; \mathbb{R}^{n}\right)$ satisfies the compatibility conditions 2.5$), 2.6$ and

$$
\left\|\xi_{o}\right\|_{H^{2}\left((0, L) ; \mathbb{R}^{n}\right)}<\varepsilon
$$

Let $\xi \in C^{2}\left(\left[0, T^{\star}\right) ; H^{2}\left((0, L) ; \mathbb{R}^{n}\right)\right)$ be the maximal classical solution of the Cauchy problem 2.1 , 2.3 and (2.4). Using implication (3.46) to (3.48) for $T \in\left[0, T^{\star}\right]$, we get that

$$
\begin{array}{r}
\|\xi(\cdot, t)\|_{H^{2}\left((0, L) ; \mathbb{R}^{n}\right)} \leq \delta_{0}, \quad \forall t \in\left[0, T^{\star}\right), \\
|\xi(\cdot, t)|_{0}+\left|\xi_{t}(t, \cdot)\right|_{0} \leq \delta, \quad \forall t \in\left[0, T^{\star}\right) .
\end{array}
$$




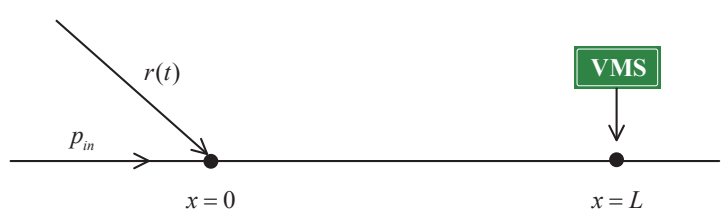

Fig. 1. A freeway section with the on-ramp and VMS.

Using 3.50 and Theorem 2.1. we have that $T=+\infty$. Using Lemma 3.6 and (3.51), we finally obtain that

$$
\|\xi(\cdot, t)\|_{H^{2}\left((0, L) ; \mathbb{R}^{n}\right)}^{2}+\zeta^{2}(t) \leq \beta V \leq \beta e^{-\alpha t} V(0) \leq \beta^{2} e^{-\alpha t}\left\|\xi_{o}\right\|_{H^{2}\left((0, L) ; \mathbb{R}^{n}\right)}^{2} .
$$

Let $C=\beta$ and $\nu=\frac{\alpha}{2}$, it follows that $\|\xi(\cdot, t)\|_{H^{2}\left((0, L) ; \mathbb{R}^{n}\right)} \leq C e^{-\nu t}\left\|\xi_{o}\right\|_{H^{2}\left((0, L) ; \mathbb{R}^{n}\right)}$.

This completes the proof of Theorem 2.3

3.4. Conditions for linear hyperbolic systems of balance laws. In order to contract the results of Theorem 2.3 with the PI boundary stabilization for the linear systems in $L^{2}$-norm, let us recall an extended result for the linearized case.

Consider the linear hyperbolic systems of balance laws

$$
\xi_{t}+\Lambda(0) \xi_{x}=M \xi, \quad t \in[0, \infty), \quad x \in(0, L),
$$

with the PI boundary feedback control

$$
\xi_{\text {in }}(t)=K_{p} \xi_{\text {out }}(t)+K_{i} \int_{0}^{t} \xi_{\text {out }}(\tau) d \tau+\bar{\theta}
$$

Take $V_{1}(\xi, \zeta)$ as the Lyapunov function for the above linearized system. By differentiating it with time along the solution of (3.53), 3.54), and using the similar techniques of Lemma 3.3, the following corollary is easily obtained.

COROLlary 3.7. 38] The linearized system (3.53), (3.54) is globally exponentially stable if there exist a diagonal matrix $P_{1} \in \mathbb{R}^{n \times n}$, a symmetric matrix $P_{2} \in \mathbb{R}^{n \times n}$, and a matrix $P_{3} \in \mathbb{R}^{n \times n}$, a real constant $\mu \in \mathbb{R}$, such that the following matrix inequalities hold, for all $x \in[0, L]$

$$
\begin{aligned}
& \text { (i) } P=\left[\begin{array}{cc}
P_{1} & P_{3} \\
\star & P_{2}
\end{array}\right]>0, \\
& \text { (ii } \left.{ }^{\prime \prime}\right) \Omega(x)=\left[\begin{array}{ccc}
\Omega_{11}^{e}(x) & \Omega_{12}^{e}(x) & P_{3}(x) \\
\star & \Omega_{22}^{e} & \Omega_{23}^{e} \\
\star & \star & \Omega_{55}^{e}
\end{array}\right]<0,
\end{aligned}
$$

where matrices $P_{3}(x), \Omega_{11}^{e}(x), \Omega_{12}^{e}(x), \Omega_{22}^{e}, \Omega_{23}^{e}$, and $\Omega_{55}^{e}$ are given as in conditions of Theorem 2.3.

Remark 3.8. In Theorem 2.3, a necessary condition is to have the spectral radii of $K_{p}$ and $K_{i}$ smaller than one. However, for the linearized case (3.53), it is only necessary to have $\Omega_{55}^{e}<0$ in the condition (ii $\left.{ }^{\prime \prime}\right)$ of Corollary 3.7. Therefore, the set of integral gains $K_{i}$ which make the nonlinear system (2.1) exponentially stable is smaller than the set of parameters obtained for the linearized system (3.53).

4. PI boundary control for ARZ traffic flow model. Boundary control of traffic flow dynamics is formulated in this section. A homogeneous freeway section including the on-ramp at the inlet boundary is sketched in Fig. 1. We assume that the on-ramp signal is regulated with the metering strategies to control the flow-rate of the driving-in vehicles into the mainline traffic, and VMSs are located at boundaries to limit the driving speed of vehicles.

As the flow-rate of driving-in vehicles from the upstream section is affected by the uncertain traffic demand, the PI boundary feedback control developed in this paper can be used to stabilize the traffic parameters (the vehicle density and speed) at the steady states. 
4.1. ARZ Traffic Flow Model. The so-called Aw-Rascle-Zhang traffic flow model is a typical quasilinear hyperbolic balance law which describes the macroscopic traffic flow dynamics as

$$
\left\{\begin{array}{l}
\partial_{t} \rho+\partial_{x}(v \rho)=0 \\
\partial_{t} v+\left(v-\rho p^{\prime}(\rho)\right) \partial_{x} v=\frac{S(\rho)-v}{\tau}
\end{array}\right.
$$

where $\rho(x, t)$ is the vehicle density, $v(x, t)$ is the average speed, $x \in(0, L)$, and $\tau$ is the relaxation time related to the driving behavior. The variable $p(\rho)$ is the traffic pressure defined as

$$
p(\rho)=v_{f}-S(\rho),
$$

where $v_{f}$ is the free flow speed. The function $S(\rho)$ is the speed-density function given by Greenshields model in [15] as

$$
S(\rho)=v_{f}\left(1-\left(\frac{\rho}{\rho_{m}}\right)^{\gamma}\right),
$$

$\rho_{m}$ is the maximum vehicle density and $\gamma>0$ is a constant.

Let $z=v, w=v+v_{f}\left(\frac{\rho}{\rho_{m}}\right)^{\gamma}$, then the ARZ equation 4.1 may be written in the characteristic Riemann coordinates as

$$
\left\{\begin{array}{l}
\partial_{t} w+z \partial_{x} w=\frac{v_{f}-w}{\tau} \\
\partial_{t} z+[(1+\gamma) z-\gamma w] \partial_{x} z=\frac{v_{f}-w}{\tau} .
\end{array}\right.
$$

In equation (4.4), the sign of the second characteristic velocity, $(1+\gamma) z-\gamma w$ being positive or negative indicates the information of vehicle speed propagating from the upstream boundary, i.e., $x=0$ to the downstream boundary, $x=L$, or contrary. This sign can be used to determine the freeway traffic lies in the free-flow or in the congested mode, as in [36. In this case, we assume that the system (4.4) is strictly hyperbolic.

Denote $\left(w^{*}, z^{*}\right)$ being the state of the system (4.4) which satisfies the relationship $w^{*}=v_{f}$. The deviations from the nominal states $\left(w^{*}, z^{*}\right)$ are defined as

$$
\begin{aligned}
& \tilde{w}(x, t)=w(x, t)-w^{*}, \\
& \tilde{z}(x, t)=z(x, t)-z^{*} .
\end{aligned}
$$

We then obtain the deviation $(\tilde{w}, \tilde{z})$-system

$$
\left\{\begin{array}{l}
\partial_{t} \tilde{w}+\left(z^{*}+\tilde{z}\right) \partial_{x} \tilde{w}=-\frac{1}{\tau} \tilde{w} \\
\partial_{t} \tilde{z}+\left[(1+\gamma) z^{*}-\gamma w^{*}+(1+\gamma) \tilde{z}-\gamma \tilde{w}\right] \partial_{x} \tilde{z}=-\frac{1}{\tau} \tilde{w}
\end{array}\right.
$$

Let $\xi=[\tilde{w}, \tilde{z}]^{\top}$, the ARZ quasilinear hyperbolic system of balance laws is given as follows:

$$
\xi_{t}+\Lambda(\xi) \xi_{x}=M \xi, \quad x \in[0, L], \quad t \in[0, \infty),
$$

in which the system matrices

$$
\Lambda(\xi)=\left[\begin{array}{cc}
z^{*}+\tilde{z} & 0 \\
0 & (1+\gamma) z^{*}-\gamma w^{*}+(1+\gamma) \tilde{z}-\gamma \tilde{w}
\end{array}\right]
$$

and

$$
M=\left[\begin{array}{ll}
-\frac{1}{\tau} & 0 \\
-\frac{1}{\tau} & 0
\end{array}\right] .
$$

4.2. PI boundary feedback control. In this subsection, using the quasilinear ARZ traffic flow model (4.7), we design the PI boundary feedback controller to stabilize the uncertain traffic demand which is regarded as the external disturbance, as the freeway traffic lies in the congested regime, i.e. $(1+\gamma) z^{*}-\gamma w^{*}<0$.

To perform the boundary feedback control, the control units are the inlet on-ramp metering $r(t)$ and the variable outlet speed limit $v(L, t)$ of the freeway section, and the measurement units are the inflow speed $v(0, t)$ 
and outflow density $\rho(L, t)$. We assume that $p_{i n}$ is the fixed traffic demand, and $\bar{p}$ is the fluctuating of the flow rate. Precisely, we introduce the PI boundary feedback law:

$$
\begin{aligned}
& r(t)=r^{*}+k_{p}^{r}\left(\rho(L, t)-\rho^{*}\right)+k_{i}^{r} \int_{0}^{t}\left(\rho(L, \tau)-\rho^{*}\right) d \tau, \\
& v(L, t)=v^{*}+k_{p}^{v}\left(v(0, t)-v^{*}\right)+k_{i}^{v} \int_{0}^{t}\left(v(0, \tau)-v^{*}\right) d \tau,
\end{aligned}
$$

where $r^{*}$ is the nominal flow rate that satisfies the equilibrium relationship $p_{i n}+r^{*}=\rho^{*} v^{*}$, and $k_{p}^{r}, k_{i}^{r}, k_{p}^{v}$ and $k_{i}^{v}$ are tuning gains.

Let $\tilde{\rho}=\rho-\rho^{*}, \tilde{v}=v-v^{*}$, and from the PI boundary controller 4.11)-4.12 directly, we have

$$
\begin{aligned}
& r(t)=r^{*}+k_{p}^{r} \tilde{\rho}(L, t)+k_{i}^{r} \int_{0}^{t} \tilde{\rho}(L, \tau) d \tau, \\
& \tilde{v}(L, t)=k_{p}^{v} \tilde{v}(0, t)+k_{i}^{v} \int_{0}^{t} \tilde{v}(0, \tau) d \tau .
\end{aligned}
$$

Using the flow-conserving condition at the inlet of the upstream boundary, i.e., $x=0$, we have

$$
p_{\text {in }}+\bar{p}+r(t)=\rho(0, t) v(0, t) .
$$

After the linearization of the flow-conserving condition 4.15 , we have the following boundary condition:

$$
\bar{p}+k_{p}^{r} \tilde{\rho}(L, t)+k_{i}^{r} \int_{0}^{t} \tilde{\rho}(L, \tau) d \tau=v^{\star} \tilde{\rho}(0, t)+\rho^{\star} \tilde{v}(0, t) .
$$

As further assume $\gamma=1$, and $a=\frac{v_{f}}{\rho_{m}}$, and deleting the high-order terms, we could rewrite the condition 4.16 in the Riemann coordinates of the system (4.7) as

$$
\begin{aligned}
\tilde{w}(0, t)= & \tilde{v}(0, t)+a \tilde{\rho}(0, t) \\
= & \frac{a \bar{p}}{v^{\star}}+\left(1-\frac{a \rho^{\star}}{v^{\star}}\right) \tilde{v}(0, t)+\frac{a k_{p}^{r}}{v^{\star}} \tilde{\rho}(L, t)+\frac{a k_{i}^{r}}{v^{\star}} \int_{0}^{t} \tilde{\rho}(L, \tau) d \tau \\
= & \frac{a \bar{p}}{v^{\star}}+\frac{k_{p}^{r}}{v^{\star}} \tilde{w}(1, t)+\frac{k_{i}^{r}}{v^{\star}} \int_{0}^{t} \tilde{w}(1, \tau) d \tau \\
& +\left(1-\frac{a \rho^{\star}}{v^{\star}}-\frac{k_{p}^{r} k_{p}^{v}}{v^{\star}}\right) \tilde{v}(0, t) \\
& -\left(\frac{k_{p}^{r} k_{i}^{v}}{v^{\star}}+\frac{k_{i}^{r} k_{p}^{v}}{v^{\star}}\right) \int_{0}^{t} \tilde{v}(0, \tau) d \tau .
\end{aligned}
$$

Combining (4.14) and 4.17) together for the system (4.7), we have the following PI boundary feedback controller

$$
\left[\begin{array}{c}
\tilde{w}(0, t) \\
\tilde{z}(L, t)
\end{array}\right]=K_{p}\left[\begin{array}{c}
\tilde{w}(L, t) \\
\tilde{z}(0, t)
\end{array}\right]+K_{i} \int_{0}^{t}\left[\begin{array}{c}
\tilde{w}(L, \tau) \\
\tilde{z}(0, \tau)
\end{array}\right] d \tau+\bar{\theta}
$$

in which the proportional and integral gains are given, respectively, as

$$
\begin{gathered}
K_{p}=\left[\begin{array}{cc}
\frac{k_{p}^{r}}{v^{\star}} & 1-\frac{a \rho^{\star}}{v^{\star}}-\frac{k_{p}^{r} k_{p}^{v}}{v^{\star}} \\
0 & k_{p}^{v}
\end{array}\right], \\
K_{i}=\left[\begin{array}{cc}
\frac{k_{i}^{r}}{v^{\star}} & \frac{-k_{p}^{r} k_{i}^{v}-k_{i}^{r} k_{p}^{v}}{v^{\star}} \\
0 & k_{i}^{v}
\end{array}\right],
\end{gathered}
$$

and the uncertain constant disturbance

$$
\bar{\theta}^{\top}=\left[\begin{array}{ll}
\frac{a \bar{p}}{v^{\star}} & 0
\end{array}\right]
$$




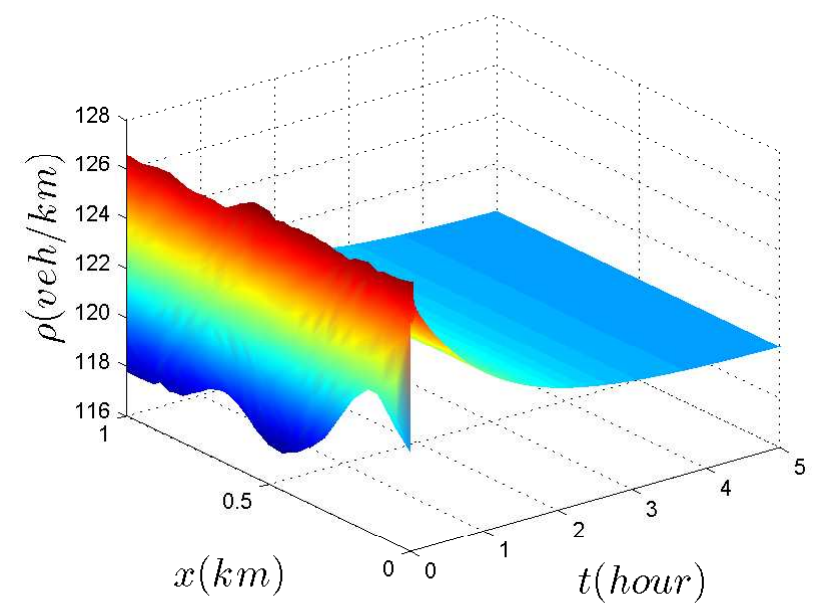
$(120,70)$.

FIG. 2. The evolution of $\rho$ of the quasilinear ARZ model (4.1) with PI boundary control (4.11)-(4.12) and state $\left(\rho^{*}, v^{*}\right)=$

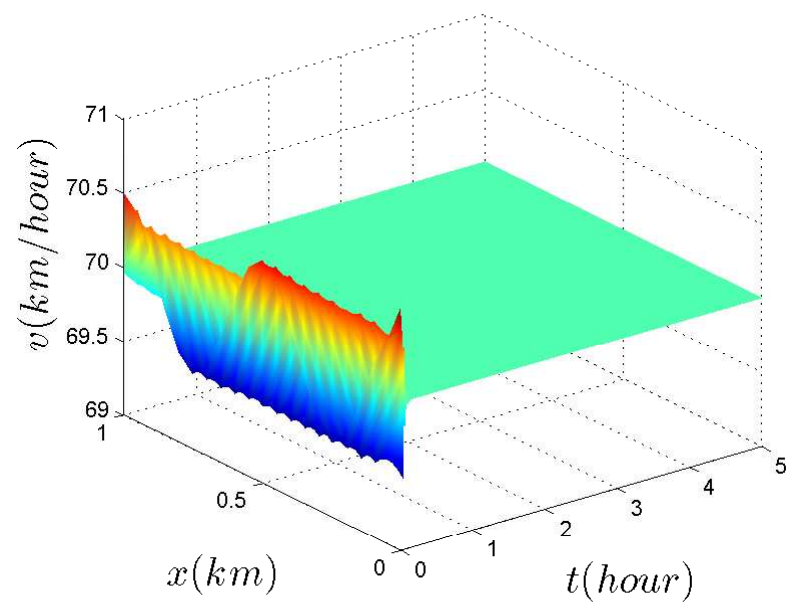

FIG. 3. The evolution of $v$ of the quasilinear ARZ model (4.1) with PI boundary control (4.11)- (4.12) and state $\left(\rho^{*}, v^{*}\right)=$ $(120,70)$.

4.3. Simulations. The developed PI boundary feedback control laws 4.11)- 4.12 for the stabilization of freeway traffic are now tested with the numerical simulations. To this end, we consider a freeway section whose road and traffic parameters are given, respectively, as $\rho_{m}=200$ veh. $/ \mathrm{km}, v_{f}=150 \mathrm{~km} /$ hour, $a=0.75$, $p_{\text {in }}=6600 \mathrm{veh} . /$ hour, $\bar{p}=420 \mathrm{veh}$./hour, and $r^{*}=1800 \mathrm{veh}$./hour. The total road length $L=1 \mathrm{~km}$ and the relation time $\tau=60$ second.

Given the state $\left(\rho^{*}, v^{*}\right)=(120,70)$, which satisfies the ARZ equations 4.7) with $\lambda_{2}^{*}=-20<0$. Choose the tuning parameters in the PI controller 4.11)-4.12 as $k_{p}^{r}=1, k_{p}^{v}=0.1, k_{i}^{r}=-65$, and $k_{i}^{v}=-0.9$, then the associated boundary condition matrices $K_{p}$, and $K_{i}$ are given, respectively, as

$$
\begin{aligned}
K_{p} & =\left[\begin{array}{cc}
0.0143 & -0.2871 \\
0 & 0.1000
\end{array}\right], \\
K_{i} & =\left[\begin{array}{cc}
-0.9286 & 0.1057 \\
0 & -0.9000
\end{array}\right] .
\end{aligned}
$$

Taking $\mu=0.01$ and solving the conditions $(i)-(i i)$ of Theorem 2.3 , we can obtain the following diagonal 


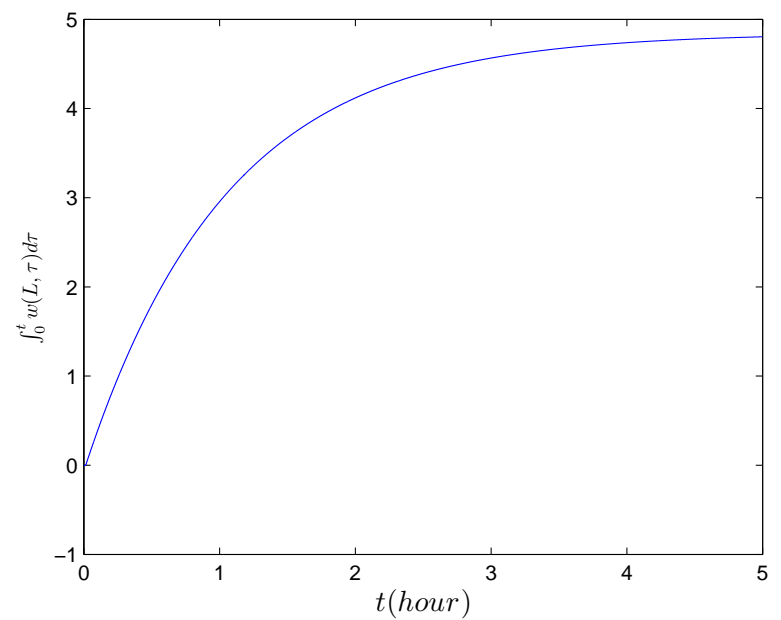

FIG. 4. The evolution of the integral of the boundary output $w(L, t)$ of the nonlinear ARZ model 44.1.).

matrix $P_{1}$, symmetric matrix $P_{2}$, and matrix $P_{3}$ as

$$
\begin{aligned}
& P_{1}=\left[\begin{array}{cc}
0.0045 & 0 \\
\star & 0.9802
\end{array}\right], \\
& P_{2}=\left[\begin{array}{cc}
0.4515 & 0.1911 \\
\star & 3.4649
\end{array}\right], \\
& P_{3}=\left[\begin{array}{ll}
0.0062 & 0.0011 \\
0.0101 & 0.1597
\end{array}\right] .
\end{aligned}
$$

To compute the numerical solutions of the system (4.7), we discretize them using the two-step variant of Lax-Wendroff method in [28]. The initial conditions from the state $\left(\rho^{*}, v^{*}\right)$ are given as

$$
\left\{\begin{array}{l}
\rho(x, 0)=\rho^{*}+0.5 \sin (4 \pi x) \\
v(x, 0)=v^{*}+1.8 \cos (4 \pi x)
\end{array} .\right.
$$

Figs. 2 and 3 show the evolution of variables, $\rho(x, t)$ and $v(x, t)$ of the nonlinear ARZ model 4.1) with the PI boundary feedback controller (4.11)-(4.12). It is observed that both converge to their state $\rho^{*}=120 \mathrm{veh} . / \mathrm{km}$, and $v^{*}=70 \mathrm{~km} /$ hour as expected from Theorem 2.3 . Fig. 4 shows the evolution of the integral of the boundary output $w(L, t)$ perturbed by the disturbance $\bar{p}$. As clearly indicated by the simulation, by virtue of the integral action in the PI controller 4.11)- 4.12 , the solutions to the ARZ model (4.1) converge to the state $\left(\rho^{*}, v^{*}\right)$ as $t \rightarrow \infty$ independently of the constant perturbations (assumed to be sufficiently small).

In contrast, we consider the linearized system at the state $\left(\rho^{*}, v^{*}\right)=(120,70)$, given as

$$
\left\{\begin{array}{l}
\partial_{t} \tilde{w}+z^{*} \partial_{x} \tilde{w}=-\frac{1}{\tau} \tilde{w} \\
\partial_{t} \tilde{z}-\left[-(1+\gamma) z^{*}+\gamma w^{*}\right] \partial_{x} \tilde{z}=-\frac{1}{\tau} \tilde{w}
\end{array}\right.
$$

with the constant characteristic velocities

$$
\lambda_{1}=z^{*}>0, \quad \lambda_{2}=-(1+\gamma) z^{*}+\gamma w^{*}>0 .
$$

For the linearized system (4.28), it is possible for us to choose the bigger integral gains, $k_{i}^{r}=-650$ and $k_{i}^{v}=-9$ than the nonlinear case of (4.22), in which these tuning parameters can not be obtained for the spacial length $L=1 \mathrm{~km}$ as stated in Remark 2.5. Then the matrix $K_{i}$ is given as

$$
K_{i}=\left[\begin{array}{cc}
-9.2857 & 1.0571 \\
0 & -9.0000
\end{array}\right] \text {. }
$$




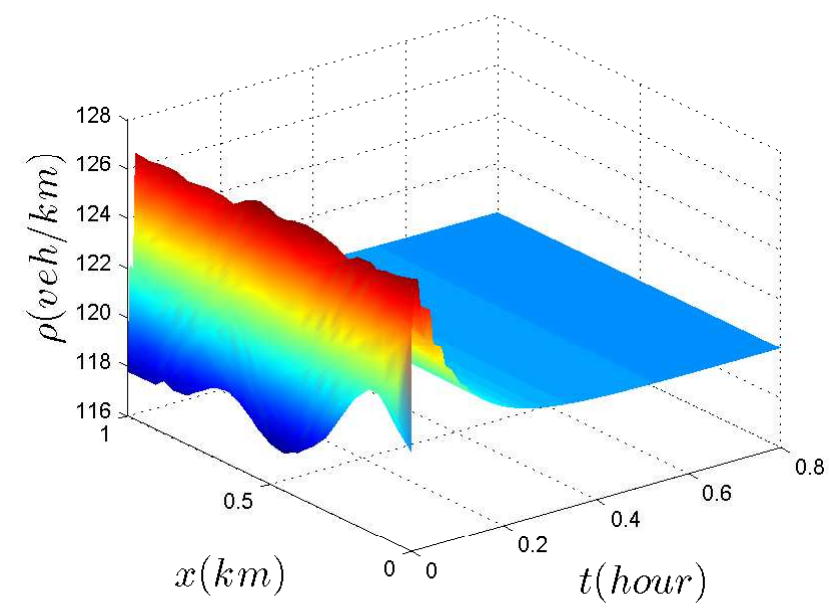
$(120,70)$

FIG. 5. The evolution of $\rho$ of the linearized ARZ model (4.28) with PI boundary control (4.11)-(4.12) and state $\left(\rho^{*}, v^{*}\right)=$

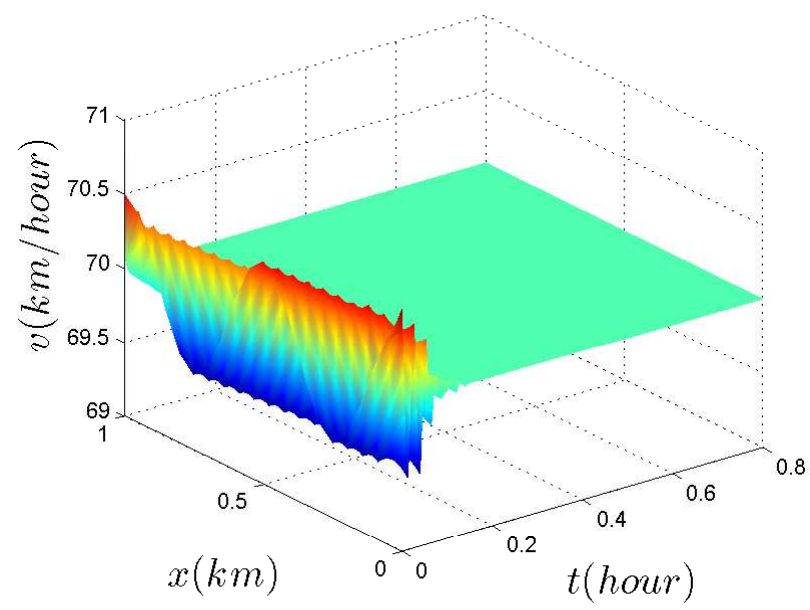

Fig. 6. The evolution of $v$ of the linearized ARZ model (4.28) with PI boundary control (4.11)-(4.12) and state $\left(\rho^{*}, v^{*}\right)=$ $(120,70)$.

Using the PI boundary controller (4.11)- 4.12 with the proportional matrix $K_{p}$ of $(4.22)$ and the new integral matrix $K_{i}$ of 4.30 , and solving the inequality conditions $(i),\left(i i^{\prime \prime}\right)$ of Corollary 3.7 for the linearized system (4.29, $\mu=0.01$, we have the following matrices

$$
\begin{aligned}
& P_{1}=\left[\begin{array}{cc}
0.0070 & 0 \\
\star & 0.0215
\end{array}\right], \\
& P_{2}=\left[\begin{array}{cc}
2.1510 & -0.4688 \\
\star & 2.8758
\end{array}\right], \\
& P_{3}=\left[\begin{array}{cc}
0.0335 & 0.0018 \\
-0.0204 & 0.1127
\end{array}\right] .
\end{aligned}
$$

Using the same initial condition (4.27) and the same constant disturbance, Figs. 5 , 6 and 7 show the evolution of the state variables of the linearized ARZ model (4.28) with the PI boundary control. It is clearly find that the closed-loop system has a faster respondence speed than the nonlinear ARZ model (4.7), as we 


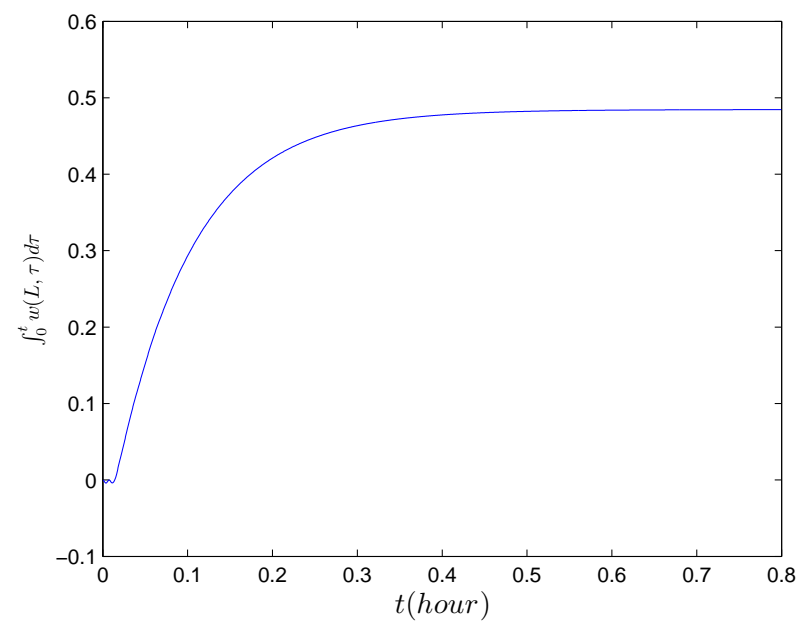

FIG. 7. The evolution of the integral of the boundary output $w(L, t)$ of the linearized ARZ model (4.28).

choose a bigger integral matrix $K_{i}$ of $(4.30)$, and almost arrive the state $\left(\rho^{*}, v^{*}\right)$ in the finite time of about 0.4 hour.

4.4. Comparison with Backstepping Method. In this subsection, we make a comparison between the Lyapunov approach and the backstepping method for the boundary control of the linearized ARZ model. The boundary control of the linear hyperbolic system of balance laws has been considered by means of the backstepping method in [8, 20]. The diagram of the control model is illustrated in Fig. 8. In this setting, only the on-ramp flux $r_{b}(t)$ is the command signal.

Applying the assumption that the downstream of the freeway section $x>L$ keeps the flux constant (see [33]), we have the boundary condition at $x=L$,

$$
\tilde{z}(L, t)=q \tilde{w}(L, t), \quad q=-\frac{\lambda_{1}}{\lambda_{2}} .
$$

Also considering the flow-conserving at the inlet of the freeway section

$$
p_{\text {in }}+\bar{p}+r_{b}(t)=\rho(0, t) v(0, t),
$$

we have the second boundary condition at $x=0$,

$$
\tilde{w}(0, t)=\frac{1}{q} \tilde{z}(0, t)+\frac{a}{v^{*}} r_{b}(t)+\frac{a \bar{p}}{v^{*}} .
$$

To get the normal form of the $2 \times 2$ linear hyperbolic systems in [8], we define the change of variables

$$
\begin{aligned}
\varepsilon^{1} & =\exp \left(\frac{x}{\tau \lambda_{1}}\right) \tilde{w}, \\
\varepsilon^{2} & =\tilde{z} .
\end{aligned}
$$

for all $x \in[0, L]$. Then the system 4.28)-4.29) and 4.34- 4.35 is transformed into the following system

$$
\begin{aligned}
& \varepsilon_{t}^{1}+\lambda_{1} \varepsilon_{x}^{1}=0, \\
& \varepsilon_{t}^{2}-\lambda_{2} \varepsilon_{x}^{2}=-\frac{1}{\tau} \exp \left(-\frac{x}{\tau \lambda_{1}}\right) \varepsilon^{1}, \\
& \varepsilon^{2}(L, t)=q \exp \left(-\frac{L}{\tau \lambda_{1}}\right) \varepsilon^{1}(L, t), \\
& \varepsilon^{1}(0, t)=\frac{1}{q} \varepsilon^{2}(0, t)+U(t)+\frac{a \bar{p}}{v^{*}},
\end{aligned}
$$




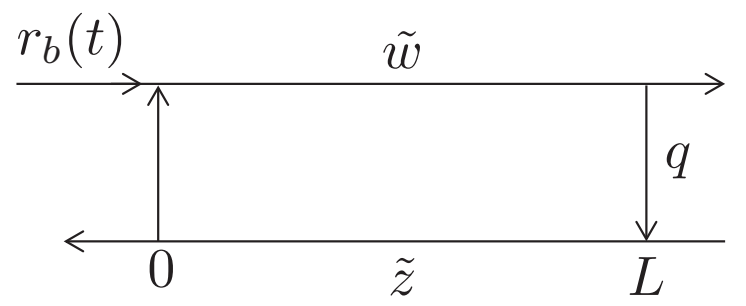

FIG. 8. Diagram of the freeway control model with the backstepping method.

where the new control variable satisfies $U(t)=\frac{a}{v^{*}} r_{b}(t)$.

In the next step, using a backstepping transformation, we map the system $4.39-4.42$ to a target system which is exponentially stable for the state $\left(\rho^{*}, v^{*}\right)$.

Let us consider the backstepping transformation

$$
\begin{aligned}
& \alpha(x, t)=\varepsilon^{1}(x, t)-\int_{x}^{L} K^{11}(x, \varsigma) \varepsilon^{1}(\varsigma, t) d \varsigma-\int_{x}^{L} K^{12}(x, \varsigma) \varepsilon^{2}(\varsigma, t) d \varsigma, \\
& \beta(x, t)=\varepsilon^{2}(x, t)-\int_{x}^{L} K^{21}(x, \varsigma) \varepsilon^{1}(\varsigma, t) d \varsigma-\int_{x}^{L} K^{22}(x, \varsigma) \varepsilon^{2}(\varsigma, t) d \varsigma,
\end{aligned}
$$

where $0 \leq \varsigma \leq x \leq L$, the kernels $K^{11}, K^{12}, K^{21}$, and $K^{22}$ are defined by a set of hyperbolic PDEs that can be found in [8].

The transformation (4.43)- 4.44 is invertible and the inverse transformation can be expressed as follow

$$
\begin{aligned}
& \varepsilon^{1}(x, t)=\alpha(x, t)+\int_{x}^{L} L^{\alpha \alpha}(x, \varsigma) \alpha(\varsigma, t) d \varsigma+\int_{x}^{L} L^{\alpha \beta}(x, \varsigma) \beta(\varsigma, t) d \varsigma, \\
& \varepsilon^{2}(x, t)=\beta(x, t)+\int_{x}^{L} L^{\beta \alpha}(x, \varsigma) \alpha(\varsigma, t) d \varsigma+\int_{x}^{L} L^{\beta \beta}(x, \varsigma) \beta(\varsigma, t) d \varsigma .
\end{aligned}
$$

where the kernels $L^{\alpha \alpha}, L^{\alpha \beta}, L^{\beta \alpha}$, and $L^{\beta \beta}$ belong to $L^{2}\left((0, L) ; \mathbb{R}^{n}\right)$.

Under the boundary feedback control law

$$
U(t)=-\frac{1}{q} \int_{0}^{L} L^{\beta \alpha}(0, \varsigma) \alpha(t, \varsigma) d \varsigma-\frac{1}{q} \int_{0}^{L} L^{\beta \beta}(0, \varsigma) \beta(t, \varsigma) d \varsigma+k_{i} \int_{0}^{t}(\beta(0, s)-\alpha(0, s)) d s,
$$

where the integral tuning parameter $k_{i} \in \mathbb{R}$, the transformation (4.43)-(4.44) maps the original system (4.39)4.42 to the following target system

$$
\begin{aligned}
& \alpha_{t}+\lambda_{1} \alpha_{x}=0, \\
& \beta_{t}-\lambda_{2} \beta_{x}=0, \\
& \beta(L, t)=q \exp \left(-\frac{L}{\tau \lambda_{1}}\right) \alpha(L, t), \\
& \alpha(0, t)=\frac{1}{q} \beta(0, t)+k_{i} \int_{0}^{t}(\beta(0, s)-\alpha(0, s)) d s+\frac{a \bar{p}}{v^{*}} .
\end{aligned}
$$

The necessary and sufficient condition (see [5, Theorem 1]) has been presented to guarantee the target system 4.48-4.51) exponentially stable by choosing the satisfied integral tuning parameter $k_{i}$.

Therefore, the original linearized ARZ model 4.28$)-(4.29)$ and $44.34-(4.35)$ is exponentially stable under the feedback control law $U(t)$. An explicit solution of the equations (4.45)-(4.46) can be found in 22. as

$$
\begin{aligned}
& L^{\alpha \alpha}(x, \varsigma)=0, \\
& L^{\alpha \beta}(x, \varsigma)=0, \\
& L^{\beta \alpha}(x, \varsigma)=\frac{1}{\tau\left(\lambda_{1}+\lambda_{2}\right)} \exp \left(-\frac{1}{\tau \lambda_{1}}\left(\frac{\lambda_{1} x+\lambda_{2} \varsigma}{\lambda_{1}+\lambda_{2}}\right)\right), \\
& L^{\beta \beta}(x, \varsigma)=\frac{q \lambda_{1}}{\tau \lambda_{2}\left(\lambda_{1}+\lambda_{2}\right)} \exp \left(-\frac{1}{\tau}\left(\frac{x-\varsigma}{\lambda_{1}+\lambda_{2}}\right)\right) .
\end{aligned}
$$




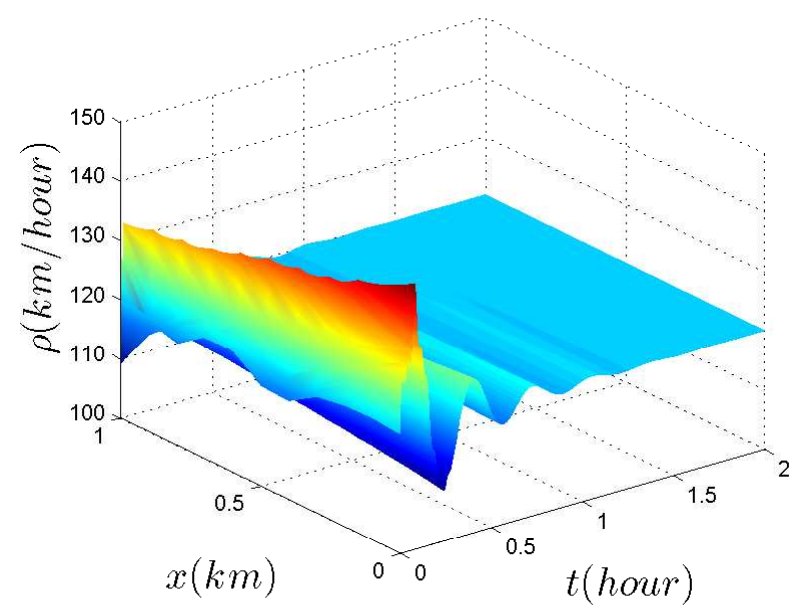
$(120,70)$

FIG. 9. The evolution of $\rho$ of the linearized ARZ model (4.28) with the backstepping control (4.47) and state $\left(\rho^{*}, v^{*}\right)=$

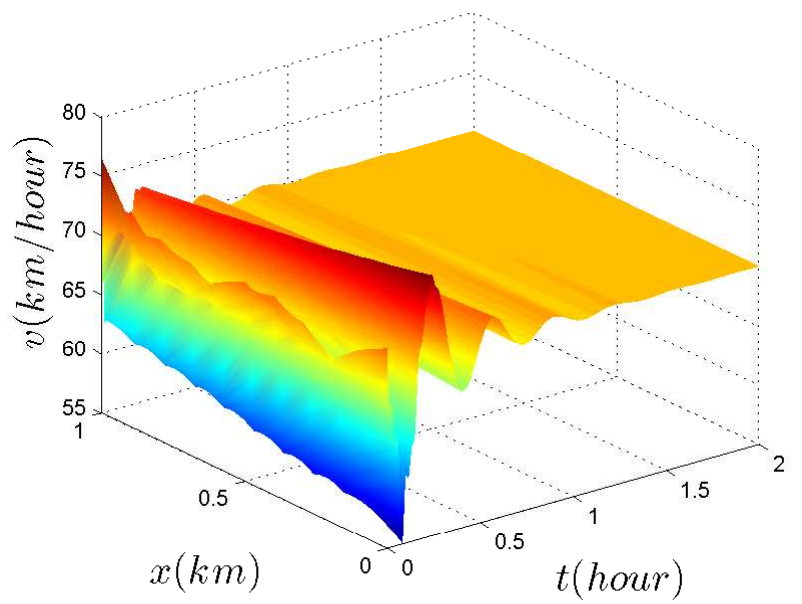

FIG. 10. The evolution of $v$ of the linearized ARZ model 4.28) with the backstepping control 4.47) and state $\left(\rho^{*}, v^{*}\right)=$ $(120,70)$.

Choosing the integral tuning parameter $k_{i}=8$, and under the same initial condition (4.27) and the same constant disturbance $\bar{p}=420$, Figs. 9 and 10 show the evolution of the state variables of the linearized ARZ model (4.28) by using the backstepping control 4.47) as displayed in Fig. 11. The closed-loop system is exponentially stable and converges to the steady state in the finite time about 1.5 hour.

Remark 4.1. As a result of the comparison, the PI boundary feedback control with the Lyapunov technique has a obvious difference with the backstepping controller which is an infinite dimensional controller indeed. The solutions of the kernel PDEs such as (4.43) and (4.44) have to be pre-computed. While, in our developed boundary feedback stabilization there are only two tuning parameter matrices selected to satisfy a set of inequalities (2.11) and (2.12). The advantages of the backstepping method can be further reviewed in the literature [21] and [20].

5. Conclusion. We have addressed the issue of PI boundary stabilization of the quasilinear hyperbolic system of balance laws in the $H^{2}$-norm to suppress the static error of boundary conditions. The main contribution is Theorem 2.3 in which the sufficient conditions are given as a set of matrix inequalities such that the classical Cauchy solution exponentially converges. The theory contribution has been illustrated with the 


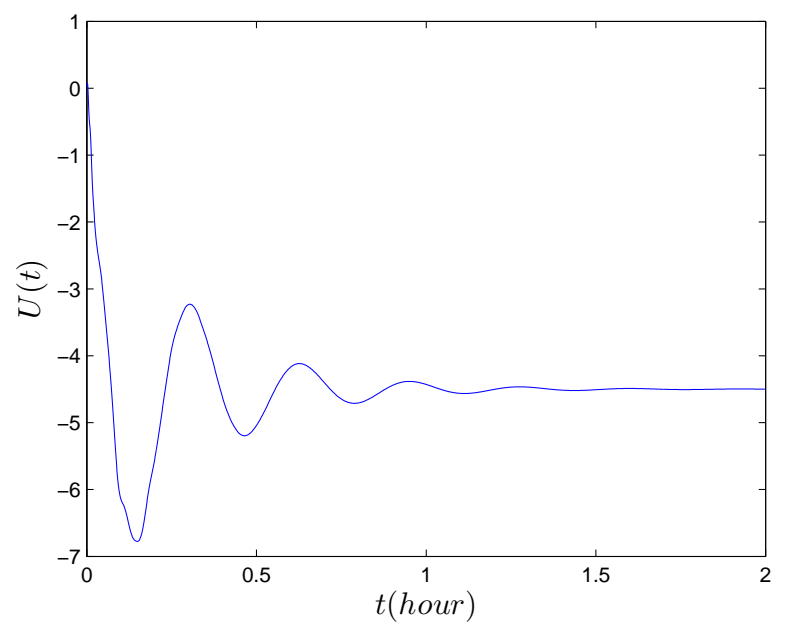

FIG. 11. The evolution of the backstepping control (4.47).

boundary control of the freeway traffic flow with uncertain traffic demand. PI boundary control by integrating on-ramp metering and variable speed limit is designed to stabilize the ARZ traffic flow model. A natural continuation of the work would be the extension to the cascade networks of freeway traffic and optimal control such as the coordinated ramp metering of [26].

A. Proof of Lemma 3.4. Recall the definition of $V_{2}$ in $\sqrt{3.3}$ and the definition of $P_{1}(x)$ in the statement of Theorem 2.3 $P_{1}(x)=P_{1} e^{-2 \mu x}$. The time derivative of $V_{2}\left(\xi_{t}\right)$ along the solutions of $\left.(3.19), 3.20\right)$ is

$$
\begin{aligned}
\dot{V}_{2}\left(\xi_{t}\right) & =2 \int_{0}^{L} \xi_{t t}^{\top} P_{1} \xi_{t} e^{-2 \mu x} d x \\
& =2 \int_{0}^{L}\left\{-\Lambda(\xi) \xi_{t x}-\operatorname{diag}\left[\frac{\partial \Lambda}{\partial \xi}(\xi) \xi_{t}\right] \xi_{x}+\frac{\partial B}{\partial \xi}(\xi) \xi_{t}\right\}^{\top} P_{1} \xi_{t} e^{-2 \mu x} d x
\end{aligned}
$$

Then, using an integration by parts, we get

$$
\dot{V}_{2}\left(\xi_{t}\right)=\mathcal{T}_{21}+\mathcal{T}_{22},
$$

with

$$
\begin{aligned}
& \mathcal{T}_{21}=\left[-\xi_{t}^{\top} \Lambda(\xi) P_{1} \xi_{t} e^{-2 \mu x}\right]_{0}^{L} \\
& \mathcal{T}_{22}=\int_{0}^{L}\left\{\xi_{t}^{\top}\left(-2 \mu \Lambda(\xi) P_{1}+\frac{\partial \Lambda}{\partial \xi}(\xi) \xi_{x} P_{1}+2 P_{1} \frac{\partial B}{\partial \xi}(\xi)\right) \xi_{t}-2 P_{1} \operatorname{diag}\left[\frac{\partial \Lambda}{\partial \xi}(\xi) \xi_{t}\right] \xi_{x}\right\} e^{-2 \mu x} d x
\end{aligned}
$$

From the boundary condition 3.20 , we have

$$
\begin{aligned}
\mathcal{T}_{21}= & -\xi_{t}^{\top}(L, t) \Lambda(0) P_{1} \xi_{t}(L, t) e^{-2 \mu L}+\left[K_{p} \xi_{t}(L, t)+K_{i} \xi_{L}(t)\right]^{\top} \Lambda(0) P_{1}\left[K_{p} \xi_{t}(L, t)+K_{i} \xi_{L}(t)\right] \\
& +\mathcal{O}\left(\left|\xi_{t}(L, t)\right|^{2}\left|\xi_{L}(t)\right|+\left|\xi_{L}(t)\right|^{2}\left|\xi_{t}(L, t)\right|+\left|\xi_{L}(t)\right|^{3} ;\left|\xi_{L}(t)\right|\right) \\
= & {\left[\begin{array}{c}
\xi_{L}(t) \\
\xi_{t}(L, t)
\end{array}\right]^{\top}\left[\begin{array}{cc}
K_{i}^{\top} \Lambda(0) P_{1} K_{i} & K_{i}^{\top} \Lambda(0) P_{1} K_{p} \\
\star & K_{p}^{\top} \Lambda(0) P_{1} K_{p}-e^{-2 \mu L} \Lambda(0) P_{1}
\end{array}\right]\left[\begin{array}{c}
\xi_{L}(t) \\
\xi_{t}(L, t)
\end{array}\right] } \\
& +\mathcal{O}\left(\left|\xi_{t}(L, t)\right|^{2}\left|\xi_{L}(t)\right|+\left|\xi_{L}(t)\right|^{2}\left|\xi_{t}(L, t)\right|+\left|\xi_{L}(t)\right|^{3} ;\left|\xi_{L}(t)\right|\right) .
\end{aligned}
$$


Moreover, noting in particular that $\int_{0}^{L} \xi_{t}^{\top} \frac{\partial \Lambda}{\partial \xi}(\xi) \xi_{x} P_{1} \xi_{t} e^{-2 \mu x} d x=\mathcal{O}\left(\int_{0}^{L}\left|\xi_{t}\right|^{2}\left|\xi_{x}\right| d x ;|\xi|_{0}\right)=\mathcal{O}\left(\int_{0}^{L}\left|\xi_{t}\right|^{3} d x ;|\xi|_{0} \mid\right)$, we get with A.3 that $\mathcal{T}_{22}$ is written as

$$
\begin{aligned}
\mathcal{T}_{22}= & \int_{0}^{L} \xi_{t}^{\top}\left[-2 \mu \Lambda(0) P_{1}+M^{\top} P_{1}+P_{1} M\right] \xi_{t} e^{-2 \mu x} d x \\
& +\mathcal{O}\left(\int_{0}^{L}\left|\xi_{t}\right|^{2}|\xi|+\left|\xi_{t}\right|^{3} d x ;|\xi|_{0}\right) .
\end{aligned}
$$

Then, $\dot{V}_{2}\left(\xi_{t}\right)$ of 3.21 is derived by combining $\mathcal{T}_{21}$ of A.4 , and $\mathcal{T}_{22}$ of A.5.

This concludes the proof of Lemma 3.4

B. Proof of Lemma 3.5. Recall the definition of $V_{3}$ in (3.4) and the definition of $P_{1}(x)$ in the statement of Theorem 2.3 $P_{1}(x)=P_{1} e^{-2 \mu x}$. The time derivative of $V_{3}\left(\xi_{t t}\right)$ along the solutions of $\left.3.22,3.23\right)$ is

$$
\begin{aligned}
\dot{V}_{3}\left(\xi_{t t}\right)= & 2 \int_{0}^{L} \xi_{t t t}^{\top} P_{1} \xi_{t t} e^{-2 \mu x} d x \\
= & 2 \int_{0}^{L}\left[-\Lambda(\xi) \xi_{t t x}-2 \operatorname{diag}\left[\frac{\partial \Lambda}{\partial \xi}(\xi) \xi_{t}\right] \xi_{t x}-\operatorname{diag}\left[\frac{\partial \Lambda}{\partial \xi}(\xi) \xi_{t}\right]_{t} \xi_{x}\right. \\
& \left.+\frac{\partial B}{\partial \xi}(\xi) \xi_{t t}+\left[\frac{\partial B}{\partial \xi}(\xi)\right]_{t} \xi_{t}\right]^{\top} P_{1} \xi_{t t} e^{-2 \mu x} d x
\end{aligned}
$$

Then, using the integration by parts, we get

$$
\dot{V}_{3}\left(\xi_{t t}\right)=\mathcal{T}_{31}+\mathcal{T}_{32}
$$

with

$$
\begin{aligned}
\mathcal{T}_{31}= & {\left[-\xi_{t t}^{\top} \Lambda(\xi) P_{1} \xi_{t t} e^{-2 \mu x}\right]_{0}^{L}, } \\
\mathcal{T}_{32}= & \int_{0}^{L} \xi_{t t}^{\top}\left[-2 \mu \Lambda(\xi) P_{1}+\frac{\partial \Lambda}{\partial \xi}(\xi) \xi_{x} P_{1}+2 P_{1} \frac{\partial B}{\partial \xi}(\xi)\right] \xi_{t t} e^{-2 \mu x} d x \\
& -2 \int_{0}^{L} \xi_{t t}^{\top} P_{1}\left\{2 \operatorname{diag}\left[\frac{\partial \Lambda}{\partial \xi}(\xi) \xi_{t}\right] \xi_{t x}+\operatorname{diag}\left[\frac{\partial \Lambda}{\partial \xi}(\xi) \xi_{t}\right]_{t} \xi_{x}-\left[\frac{\partial B}{\partial \xi}(\xi)\right]_{t} \xi_{t}\right\} e^{-2 \mu x} d x .
\end{aligned}
$$

From 3.23 , we have

$$
\begin{aligned}
\mathcal{T}_{31}= & -\xi_{t t}^{\top}(L, t) \Lambda(0) P_{1} \xi_{t t}(L, t) e^{-2 \mu L}+\left[K_{p} \xi_{t t}(L, t)+K_{i} \xi_{t}(L, t)\right]^{\top} \Lambda(0) P_{1}\left[K_{p} \xi_{t t}(L, t)+K_{i} \xi_{t}(L, t)\right] \\
& +\mathcal{O}\left(\left|\xi_{t t}(L, t)\right|^{2}\left|\xi_{L}(t)\right|+\left|\xi_{t}(L, t)\right|^{2}\left|\xi_{L}(t)\right|+\left|\xi_{t t}(L, t)\right|\left|\xi_{t}(L, t)\right|\left|\xi_{L}(t)\right| ;\left|\xi_{L}(t)\right|\right) \\
= & {\left[\begin{array}{c}
\xi_{t}(L, t) \\
\xi_{t t}(L, t)
\end{array}\right]^{\top}\left[\begin{array}{cc}
K_{i}^{\top} \Lambda(0) P_{1} K_{i} & K_{i}^{\top} \Lambda(0) P_{1} K_{p} \\
\star & K_{p}^{\top} \Lambda(0) P_{1} K_{p}-e^{-2 \mu L} \Lambda(0) P_{1}
\end{array}\right]\left[\begin{array}{c}
\xi_{t}(L, t) \\
\xi_{t t}(L, t)
\end{array}\right] } \\
& +\mathcal{O}\left(\left|\xi_{t t}(L, t)\right|^{2}\left|\xi_{L}(t)\right|+\left|\xi_{t}(L, t)\right|^{2}\left|\xi_{L}(t)\right|+\left|\xi_{t t}(L, t)\right|\left|\xi_{t}(L, t)\right|\left|\xi_{L}(t)\right| ;\left|\xi_{L}(t)\right|\right) .
\end{aligned}
$$

Moreover, noting in particular that $\int_{0}^{L} \xi_{t t}^{\top} P_{1}\left\{2 \operatorname{diag}\left[\frac{\partial \Lambda}{\partial \xi}(\xi) \xi_{t}\right] \xi_{t x}\right\} e^{-2 \mu x} d x=\mathcal{O}\left(\int_{0}^{L}\left|\xi_{t t}\right|\left|\xi_{t}\right|\left|\xi_{t x}\right| d x ;|\xi|_{0}\right)=$ $\mathcal{O}\left(\int_{0}^{L}\left|\xi_{t t}\right|^{2}\left|\xi_{t}\right| d x ;|\xi|_{0} \mid\right)$, we get with $\mathrm{B} .3$ that $\mathcal{T}_{32}$ is written as

$$
\begin{aligned}
\mathcal{T}_{32}= & \int_{0}^{L} \xi_{t t}^{\top}\left[M^{\top} P_{1}+P_{1} M-2 \mu \Lambda(0) P_{1}\right] \xi_{t t} e^{-2 \mu x} d x \\
& +\mathcal{O}\left(\int_{0}^{L}\left(\left|\xi_{t t}\right|^{2}|\xi|+\left|\xi_{t t}\right|^{2}\left|\xi_{t}\right|+\left|\xi_{t t}\right|\left|\xi_{t}\right|^{2}\right) d x ;\left|\xi_{t}\right|_{0}+|\xi|_{0}\right) .
\end{aligned}
$$

Then, $\dot{V}_{3}\left(\xi_{t t}\right)$ of 3.24 is derived by combining $\mathcal{T}_{31}$ of B.4 , and $\mathcal{T}_{32}$ of B.5.

This concludes the proof of Lemma 3.5 


\section{REFERENCES}

[1] A. Aw and M. Rascle. Resurrection of 'second order' models of traffic flow. SIAM Journal on Applied Mathematics, 60(3):916-938, 2000.

[2] F. Bakhshande and D. Soffker. Robust control approach for a hydraulic differential cylinder system using a proportionalintegral-observer-based backstepping control. In Proceedings of the American Control Conference, Seattle, United States, 2017.

[3] G. Bastin and J. M. Coron. On boundary feedback stabilization of non-uniform linear $2 \times 2$ hyperbolic systems over a bounded interval. Systems \& Control Letters, 60(11):190-196, 2011.

[4] G. Bastin and J.-M. Coron. Stability and Boundary Stabilization of 1-D Hyperbolic Systems. Progress in Nonlinear Differential Equations and Their Applications. Springer, 2016.

[5] G. Bastin, J.-M. Coron, and S. O. Tamasoiu. Stability of linear density-flow hyperbolic systems under PI boundary control. Automatica, 53(1):37-42, 2015.

[6] F. Belletti, M. Huo, X. Litrico, and A. M. Bayen. Prediction of traffic convective instability with spectral analysis of the aw-rascle-zhang model. Physics Letters A, 379(38):2319-2330, 2015.

[7] J.-M. Coron, G. Bastin, and B. d'Andréa Novel. Dissipative boundary conditions for one-dimensional nonlinear hyperbolic systems. SIAM Journal on Control and Optimization, 47(3):1460-1498, 2008.

[8] J.-M. Coron, R. Vazquez, M. Krstic, and G. Bastin. Local exponential $H^{2}$ stabilization of a $2 \times 2$ quasilinear hyperbolic system using backstepping. SIAM Journal on Control and Optimization, 51(3):2005-2035, 2013.

[9] C. F. Daganzo. Requiem for second-order fluid approximations of traffic flow. Transportation Research Part B: Methodological, 29(4):277-286, 1995.

[10] M. Diagne, N. Bekiaris-Liberis, and M. Krstic. Time- and state-dependent input delay-compensated bang-bang control of a screw extruder for 3D printing. International Journal of Robust and Nonlinear Control, 27(17):3727-3757, 2017.

[11] V. Dos Santos, G. Bastin, J.-M. Coron, and B. d'Andréa Novel. Boundary control with integral action for hyperbolic systems of conservation laws: stability and experiments. Automatica, 44(5):1310-1318, 2008.

[12] V. Dos Santos and C. Prieur. Boundary control of open channels with numerical and experimental validations. IEEE Transactions on Control Systems Technology, 16(6):1252-1264, 2008.

[13] P. W. Dower and P. M. Farrel. On linear control of backward pumped Raman amplifiers. In IFAC Symposium on System Identification, pages 547-552, Newcastle, Australia, 2006.

[14] J. M. Greenberg and T. T. Li. The effect of boundary damping for the quasilinear wave equations. Journal of Differential Equations, 52:66-75, 1984

[15] B. D. Greenshields. A study of traffic capacity. In Proceedings of the Highway Research Board, pages 448-477, 1935.

[16] M. Gugat. Exponential stabilization of the wave equation by Dirichlet integral feedback. SIAM Journal on Control and Optimization, 53(1):526-546, 2015.

[17] M. Gugat. Neumann boundary feedback stabilization for a nonlinear wave equation: A strict $H^{2}$-Lyapunov function. Mathematical Control and Related Fields, 7(3):419-448, 2017.

[18] M. Gugat, G. Leugering, S. Tamasou, and K. Wang. $H^{2}$-stabilization of the isothermal Euler equations: a Lyapunov function approach. Chinese Annals of Mathematics, Series B, 33(4):479-500, 2012.

[19] M. Herty and W-A. Yong. Feedback boundary control of linear hyperbolic systems with relaxation. Automatica, 69:12-17, 2016.

[20] L. Hu, R. Vazquez, D. Meglio, and M. Krstic. Boundary exponential stabilization of 1-D inhomogeneous quasilinear hyperbolic systems. Technical Report arXiv:1512:03539, preprint, 2015.

[21] M. Krstic and A. Smyshlyaev. Boundary Control of PDEs: A Course on Backstepping Designs. SIAM, Philadelphia, PA, USA, 2008.

[22] P. O. Lamare and N. Bekiaris-Liberis. Control of $2 \times 2$ linear hyperbolic systems: Backstepping-based trajectory generation and PI-based tracking. Systems \&5 Control Letters, 86:24-33, 2015.

[23] M. J. Lighthill and J. B. Whitham. On kinematic waves. ii. a theory of traffic flow on long crowded roads. Proc. R. Soc. A, pages 317-345, 1955.

[24] F. Lutscher and A. Stevens. Emerging patterns in a hyperbolic model for locally interacting cell systems. Journal of Nonlinear Science, 12:619-640, 2002.

[25] A. Pazy. Semigroups of linear operators and applications to partial differential equations. Appl. Math. Sci. 44. SpringerVerlag, New York, 1983.

[26] J. Reilly, S. Samaranayake, M.L.D. Monache, W. Krichene, P. Goatin, and A.M. Bayen. Adjoint-based optimization on a network of discretized scalar conservation laws with applications to coordinated ramp metering. Journal of Optimization Theory and Applications, 167(2):733-760, 2015.

[27] P. I. Richards. Shock waves on the highway. Oper. Res., pages 42-51, 1956.

[28] L.F. Shampine. Solving hyperbolic PDEs in Matlab. Appl. Numer. Anal. \& C Comput. Math., 2:346-358, 2005.

[29] A. Terrand-Jeanne, V. Andrieu, V. Dos Santos Martins, and C.-Z. Xu. Lyapunov functionals for output regulation of exponentially stable semi-groups via integral action and application to PDE hyperbolic systems. In Proceedings of IEEE 57th Conference on Decisionand Control, Miami Beach, United States, 2018.

[30] N. T. Trinh, V. Andrieu, and C.-Z. Xu. Design of integral controllers for nonlinear systems governed by scalar hyperbolic partial differential equations. IEEE Transactions on Automatic Control, 62(9):4527-4536, 2017.

[31] N. T. Trinh, V. Andrieu, and C.-Z. Xu. Output regulation for a cascaded network of $2 \times 2$ hyperbolic systems with PI controller. Automatica, 91:270-278, 2018.

[32] C.-Z. Xu and G. Sallet. Multivariable boundary PI control and regulation of a fluid flow system. Math. Control Relat. Fields, 4:501-520, 2014

[33] H. Yu and M. Krstic. Traffic congestion control for Aw-Rascle-Zhang model. Automatica, 100:38-51, 2018

[34] H. M. Zhang. A non-equilibrium traffic model devoid of gas-like behavior. Transportation Research Part B, 36(3):275-290, 
2002.

[35] L. Zhang and C. Prieur. Necessary and sufficient conditions on the exponential stability of positive hyperbolic systems. IEEE Transactions on Automatic Control, 62(7):3610-3617, 2017.

[36] L. Zhang and C. Prieur. Stochastic stability of markov jump hyperbolic systems with application to traffic flow control. Automatica, 86:29-37, 2017.

[37] L. Zhang, C. Prieur, and J. Qiao. Local exponential stabilization of semi-linear hyperbolic systems by means of a boundary feedback control. IEEE Control Systems Letters, 2(1):55-60, 2018.

[38] L. Zhang, C. Prieur, and J. Qiao. PI boundary control of linear hyperbolic balance laws with stabilization of ARZ traffic flow models. Systems \& Control Letters, 123:85-91, 2019. 